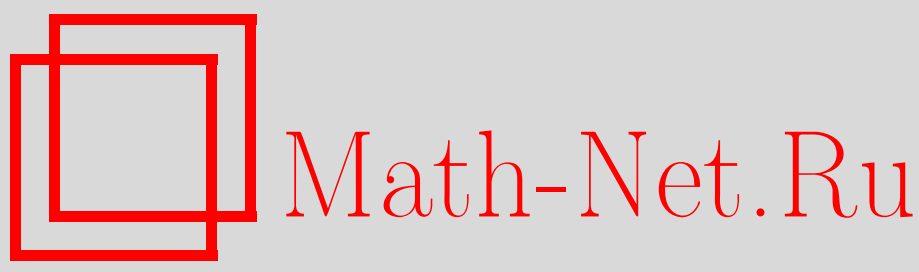

В. И. Лебедев, Экстремальные многочлены и методы оптимизации вычислительных алгоритмов, Матем. сб., 2004, том 195, номер 10, 21-66

DOI: https://doi.org/10.4213/sm852

Использование Общероссийского математического портала Math-Net.Ru подразумевает, что вы прочитали и согласны с пользовательским соглашением

http://www . mathnet.ru/rus/agreement

Параметры загрузки:

IP: 3.95 .254 .165

26 апреля 2023 г., $11: 18: 02$ 
УДК $517.518 .8+519.6$

\title{
В.И. Лебедев
}

\section{Экстремальные многочлены и методы оптимизации вычислительных алгоритмов}

\begin{abstract}
В статье изучены экстремальные на $[-1,1]$ многочлены Чебышёва-Маркова-Бернштейна-Сегё $C_{n}(x)$ с весовыми функциями $w(x)=(1+x)^{\alpha} \times$ $(1-x)^{\beta} / \sqrt{S_{l}(x)}$, где $\alpha, \beta=0, \frac{1}{2}, S_{l}(x)=\prod_{k=1}^{m}\left(1-c_{k} T_{l_{k}}(x)\right)>0$. Дана единая формула их представления в тригонометрическом виде. Получены оптимальные распределения узлов взвешенной интерполяции, явные квадратурные формулы типа Гаусса, Маркова, Лобатто, Радо для интегралов с весом $p(x)=$ $w^{2}(x)\left(1-x^{2}\right)^{-1 / 2}$. Определены параметры чебьшёвских итерационных методов, оптимально уменшшаюих ошибку по сравнению с начальной ошибкой, заданных в различных нормах. Для каждого уровня метода Федоренко-Бахвалова найдены итерационные параметры, учитывающие резултаты предыдущих вычислений. Построены чебышёвские с весом фильтры. Исследованы итерационные методы решения уравнений с компактными операторами.

Библиография: 45 названий.
\end{abstract}

\section{Введение}

Построение многих оптимальных алгоритмов вычислительной математики основано на использовании свойств многочленов, которые в произведении с некоторой весовой функцией наименее отклоняются от нуля. Применение зависящих от параметров весовых функций позволяет более точно учесть априорную информацию о свойствах класса искомых решений. На подобных методах базируется исследование в статье таких задач, как задача о распределении $n$ узлов интерполяции, даюшем наилучшее взвешенное на отрезке приближение с константой Лебега $O(\ln n)$, получение явных формул для параметров квадратурных формул повышенной точности типа Гаусса, Лобатто, Радо, Маркова, точных для интегралов с чебьшёвскими весами от дробно-рациональных функций, определение параметров чебышёвских итерационных методов, оптимально уменњшаюших ошибку по сравнению с начальной ошибкой, если начальная и конечная ошибки заданы в различных полиномиальных нормах, использование в чебышёвских с весом фильтрах для обработки сигналов и улучшения характеристик явных и неявных разностных схем.

Сформулируем две типичные возникаюшие при этом экстремальные задачи. Пусть функция $w(x)=w(x, C)$ непрерывна по $x$ на $[-1,1]$, положительна в $(-1,1)$ и является непрерывной функцией от параметров $C=\left(c_{1}, c_{2}, \ldots, c_{m}\right)$ в некоторой

Работа выполнена при финансовой поддержке Российского фонда фундаментальньтх исследований (гранты № № 02-01-00651, 02-01-00742).

(C) В. И. ЛЕведев 2004 
замкнутой области их изменения $\bar{C}$. Пусть $\Pi_{n}-$ класс многочленов $P_{n}(x) n$-й степени, нормированных каким-либо дополнительным условием, например с фиксированным коэффициентом $\widetilde{A}_{n}$ при $x^{n}$ или с заданным значением в некоторой точке $\delta>1, \bar{x}_{n}=\left(x_{1}, \ldots, x_{n}\right)$ - корни многочлена $P_{n}(x)$. Требуется найти решения следующих экстремальных задач.

ЗАДАчА I. При заданном значении $C$ найти

$$
\begin{aligned}
e_{n} & =\min _{Q_{n}(x) \in \Pi_{n}} \max _{x \in[-1,1]}\left|Q_{n}(x) w(x, C)\right|, \\
P_{n}(x) & =\arg \min _{Q_{n}(x) \in \Pi_{n}} \max _{x \in[-1,1]}\left|Q_{n}(x) w(x, C)\right| .
\end{aligned}
$$

Многочлены $P_{n}(x)$ назовем многочленами, наименее отклоняющимися на $[-1,1]$ от нуля с весом $w(x)$, или әкстремальныци (с весом) многочленами.

ЗАДАчА II. Найти

$$
\begin{aligned}
e_{n} & =\min _{C \in \bar{C}} \inf _{Q_{n}(x) \in \Pi_{n}} \max _{x \in[-1,1]}\left|Q_{n}(x) w(x, C)\right|, \\
P_{n}(x) & =\arg \min _{C \in \bar{C}} \inf _{Q_{n}(x) \in \Pi_{n}} \max _{x \in[-1,1]}\left|Q_{n}(x) w(x, C)\right| .
\end{aligned}
$$

Известно, что использование свойств чебьшёвских многочленов лежит в основе построения оптимальных алгоритмов в теории приближений, интерполирования, численного интегрирования, итерационных методов на достаточно широких классах решений [1]-[3]. Укажем четыре исследованных Чебышёвым замечательных случая экстремальных многочленов - решений задачи I. Если $x=\cos \theta$, $w(x)=w(x, C)$, а $p(x)$ - вес, с которым многочлены ортогональны на отрезке $[-1,1]$, то многочлены Чебышёва $1-4$ родов получаются:

- 1 рода при $w(x) \equiv 1$; тогда

$$
\begin{aligned}
& p(x)=\frac{1}{\sqrt{1-x^{2}}}, \quad P_{n}(x)=T_{n}(x)=\frac{1}{2}\left(\left(x+\sqrt{x^{2}-1}\right)^{n}+\left(x-\sqrt{x^{2}-1}\right)^{n}\right)=\cos n \theta ; \\
& -2 \text { рода при } w(x)=\sqrt{1-x^{2}} ; \text { тогда } \\
& \quad p(x)=\sqrt{1-x^{2}}, \quad P_{n}(x)=U_{n}(x)=\frac{\sin ((n+1) \theta)}{\sin \theta} ; \\
& -3 \text { рода при } w(x)=\sqrt{1+x} ; \text { тогда }
\end{aligned}
$$

$$
p(x)=\sqrt{\frac{1+x}{1-x}}, \quad P_{n}(x)=V_{n}(x)=2^{-1 / 2} \frac{\cos ((n+1 / 2) \theta)}{\cos (\theta / 2)} ;
$$

-4 рода при $w(x)=\sqrt{1-x}$; тогда

$$
p(x)=\sqrt{\frac{1-x}{1+x}}, \quad P_{n}(x)=W_{n}(x)=2^{-1 / 2} \frac{\sin ((n+1 / 2) \theta)}{\sin (\theta / 2)} .
$$


Эти многочлены имеют норму равную $\sqrt{\pi / 2}$, и $e_{n}=1$ при $n \geqslant 1$. Четьре группы формул можно записать единьм образом. Пусть $\alpha, \beta=0, \frac{1}{2}, N=n+\alpha+\beta$, тогда при

$$
\begin{gathered}
w(x)=w(x, \alpha, \beta)=(1+x)^{\alpha}(1-x)^{\beta} ; \\
p(x)=p(x, \alpha, \beta)=w^{2}(x, \alpha, \beta)(1+x)^{-1 / 2}(1-x)^{-1 / 2} \\
P_{n}(x)=\frac{\cos (N \theta-\beta \pi)}{w(x, \alpha, \beta)}, \quad x_{i}=\cos \frac{(i+\beta-1 / 2) \pi}{N}, \quad i=\overline{1, n},
\end{gathered}
$$

С помощью добавления к аргументу под знаком косинуса фазовой функции $\psi(\theta)$ в статье будут даны обобшения формул $(0.5)$ для $P_{n}(x)($ см. $(1.5))$, названных многочленами Чебьшёва-Маркова-Бернштейна-Сегё [4]-[9]. Для более широкого класса функций $w(x, C)($ см. $(1.1),(1.4))$ будут изучены свойства этих многочленов. Будет показано, что, имея дополнительную информацию о решении, можно с помощью упомянутых многочленов улучшить параметры многих оптимальных методов. Конспективное содержание результатов статьи содержится в [10]-[12]; она является расширенньм содержанием лекций [13]. Автор благодарен В. А. Клячину, В.И. Костенкову, К.В. Ушакову, Д.В. Чижикову за помощь в оформлении статьи.

\section{$\S 1$. О единой формуле для фазовой функции экстремальных ЧМБС-многочленов 1-4 родов}

В основе исследования задачи II лежит решение задачи I, аналитическое решение которой редко известно. Однако существует класс весовых функций, всюду плотный в конусе ограниченных непрерывных положительных в $(-1,1)$ функций, экстремальные многочлены для которых определяются простьми аналитическими формулами. Это класс весовых функций вида

$$
w(x)=w(x, \alpha, \beta, C)=\frac{(1+x)^{\alpha}(1-x)^{\beta}}{\sqrt{S_{l}(x)}}
$$

где $S_{l}(x)$ - положительный на $[-1,1]$ многочлен степени $l$, коэффициенты, или корни, или степень которого могут играть роль параметров $c_{i}$, а $\alpha, \beta=0, \frac{1}{2}$.

Пусть $Z$ и $H$ - плоскости комплексных переменных соответственно $z=x+i y$ и $h$. Рассмотрим преобразование

$$
z=\frac{1}{2}\left(h^{2}+\frac{1}{h^{2}}\right)
$$

За области однолистности отображения возьмем квадранты $D_{1}(h: \operatorname{Re} h>0$, $\operatorname{Im} h>0), D_{2}(h: \operatorname{Re} h<0, \operatorname{Im} h>0), D_{3}(h: \operatorname{Re} h<0, \operatorname{Im} h<0)$, $D_{4}(h: \operatorname{Re} h>0, \operatorname{Im} h<0)$. Каждая область $D_{k}$ отображается на $Z_{k}$ плоскости комплексного переменного с разрезами по оси $x$ от 1 до $\infty$ и от -1 до $-\infty$. Построим риманову поверхность $R$ для обратного преобразования $h(z)$, которое является четырехзначньм. Для этого склеим с учетом непрерывности отображения листы $Z_{1}$ 
с $Z_{4}$ и $Z_{2}$ с $Z_{3}$ на разрезе от 1 до $\infty$ и листы $Z_{1}$ с $Z_{2}$ и $Z_{3}$ с $Z_{4}$ на разрезе от -1 до $-\infty$. Тогда для $z \in R$

$$
h(z)=\sqrt{\frac{z+1}{2}}+\sqrt{\frac{z-1}{2}} .
$$

Использование преобразования (1.2) вместо преобразования Жуковского позволит единым методом получать формулы через степени от $h(z)$ для классических чебышёвских многочленов и экстремальных многочленов любого рода.

Пусть далее $\alpha, \beta=0, \frac{1}{2}, N=n+\alpha+\beta, \nu=N-\frac{l}{2}, x=\cos \theta, 0 \leqslant \operatorname{Re} \theta \leqslant \pi$, $\theta_{k}^{0}=\left(k+\beta-\frac{1}{2}\right) \pi / N$. Для простоты изложения будем считать, что $\nu>0$, хотя некоторые формулы будут справедливы и при $\nu=0$.

Оказалось удобньм использовать следуюшую форму для многочлена $S_{l}(x)$ [11]-[14]:

$$
S_{l}(x)=\prod_{k=1}^{m}\left(1-c_{k} T_{l_{k}}(x)\right)
$$

где $l_{1}, l_{2}, \ldots, l_{m}$ - положительные целые числа, $l=\sum_{k=1}^{m} l_{k}$, а $c_{1}, c_{2}, \ldots, c_{m}-$ вообше говоря, комплексные числа такие, что $c_{j}^{2} \notin[1, \infty) \cup\{0\}$ и если $\operatorname{Im} c_{j} \neq 0$, то в представлении (1.4) сушествует и число $c_{k}=\bar{c}_{j}$ с $l_{k}=l_{j}\left(S_{0}(x) \equiv 1\right)$. В работе Маркова [5] рассмотрен случай с $l_{k}=1, \alpha=\beta=0$ и с четньм $m$.

Далее покажем, что решением задачи I (0.1) с условиями (1.1), (1.4) с точностью до нормировочного множителя будет экстремальный с весом $w(x, \alpha, \beta, C)$ на $[-1,1]$ многочлен $C_{n}(x)$, определенный формулой

$$
C_{n}(x)=C_{n}(x, \alpha, \beta, C)=\frac{\cos (N \theta-\beta \pi+\psi(\theta))}{w(x, \alpha, \beta, C)},
$$

где $\psi(\theta)$ - зависящая только от $C$ искомая функция, назовем ее фазовой функциeй. Эти многочлены при $N \geqslant \frac{l}{2}$ обобшают соответствуюшие многочлены Чебышёва $1-4$ родов (см. (0.5) при $\left.S_{l}(x) \equiv 1\right)$. Поскольку в работах Чебышёва, Маркова, Бернштейна, Сегё [4]-[9] было положено начало исследованиям свойств таких экстремальных многочленов, назовем их ЧМБС-многочленами [14]-[17]. Авторы этих работ использовали различные представления для многочлена $S_{l}(x)$ и получили различные свои формулы для ЧМБС-многочленов отдельно для каждого рода (при $\alpha=\beta=0$ - 1-го, $\alpha=\beta=\frac{1}{2}-2$-го, $\alpha=\frac{1}{2}, \beta=0$ - 3 -го, $\alpha=0, \beta=\frac{1}{2}$ - 4-го рода).

Определим функцию $\psi(\theta)$ как сумму:

$$
\psi(\theta)=\sum_{k=1}^{m} \psi_{k}(\theta)
$$

где каждую непрерывную функцию $\psi_{k}(\theta)$ зададим соотношениями

$$
\exp \left(i\left(\frac{l_{k} \theta}{2}+\psi_{k}(\theta)\right)\right)=\frac{1}{\sqrt{1-c_{k} \cos \left(l_{k} \theta\right)}}\left(\sqrt{1-c_{k}} \cos \frac{l_{k} \theta}{2}+i \sqrt{1+c_{k}} \sin \frac{l_{k} \theta}{2}\right) .
$$


Из (1.7) следует, что

$$
\operatorname{tg}\left(\frac{l_{k} \theta}{2}+\psi_{k}(\theta)\right)=\sqrt{\frac{1+c_{k}}{1-c_{k}}} \operatorname{tg} \frac{l_{k} \theta}{2}, \quad k=\overline{1, m} .
$$

Из этих уравнений находим $\psi_{k}(\theta)$. Для действительных $c_{k} \in(-1,1)$

$$
\psi_{k}(\theta)=\operatorname{arctg} \frac{\sin \left(l_{k} \theta\right)}{A_{k}-\cos \left(l_{k} \theta\right)}, \quad \psi_{k}(0)=\psi_{k}(\pi)=0,
$$

где $A_{k}=c_{k}^{-1}+\sqrt{c_{k}^{-2}-1},\left|A_{k}\right| \geqslant 1$. Здесь выбрана та непрерывная ветвь $\operatorname{arctg}$, для которой $\operatorname{arctg} 0=0$.

Если $c_{k}$ и $c_{j}$ комплексно сопряжены, то

$$
\begin{aligned}
\psi_{j}(\theta)+\psi_{k}(\theta)= & \operatorname{arctg} \frac{\sin \left(l_{k} \theta\right)\left(A_{k}+\bar{A}_{k}-2 \cos \left(l_{k} \theta\right)\right)}{2 \cos ^{2}\left(l_{k} \theta\right)-\left(A_{k}+\bar{A}_{k}\right) \cos \left(l_{k} \theta\right)+\left|A_{k}\right|^{2}-1}, \\
& \psi_{j}(0)+\psi_{k}(0)=\psi_{j}(\pi)+\psi_{k}(\pi)=0 .
\end{aligned}
$$

Тогда

$$
\psi(0)=\psi(\pi)=0 .
$$

Производная $\psi^{\prime}(\theta)$ определена формулой

$$
\psi^{\prime}(\theta)=\frac{1}{2} \sum_{k=1}^{m} l_{k}\left(\frac{\sqrt{1-c_{k}^{2}}}{1-c_{k} \cos \left(l_{k} \theta\right)}-1\right) .
$$

Для слагаемых $\psi_{k}^{\prime}(\theta)$ в ней при действительных $c_{k} \in(-1,1)$ справедливы двусторонние оценки

$$
\frac{l_{k}}{2}\left(\sqrt{\frac{1-\left|c_{k}\right|}{1+\left|c_{k}\right|}}-1\right) \leqslant \psi_{k}^{\prime}(\theta) \leqslant \frac{l_{k}}{2}\left(\sqrt{\frac{1+\left|c_{k}\right|}{1-\left|c_{k}\right|}}-1\right),
$$

а в [16] получены оценки для $\left|\psi_{j}^{\prime}(\theta)+\psi_{k}^{\prime}(\theta)\right|$, если $c_{k}=\bar{c}_{j}$ и комплексны.

Пусть $\nu=N-\frac{l}{2}-$ целое или полуцелое число, определим ветвь функции $h(z)(1.3)$ и $h^{-1}(z)$ формулами

$$
h^{ \pm 1}(x)=\sqrt{\frac{x+1}{2}} \pm \sqrt{\frac{x-1}{2}}=\exp \left( \pm \frac{i \theta}{2}\right) .
$$

Используя (1.7), получаем

$$
\begin{aligned}
\cos (N \theta-\beta \pi+\psi(\theta))=\cos \left(\nu \theta-\beta \pi+\frac{l \theta}{2}+\psi(\theta)\right) \\
=\frac{1}{2}\left(\exp (i(\nu \theta-\beta \pi)) \prod_{k=1}^{m} \exp \left(i\left(\frac{l_{k} \theta}{2}+\psi_{k}(\theta)\right)\right)\right. \\
\left.\quad+\exp (-i(\nu \theta-\beta \pi)) \prod_{k=1}^{m} \exp \left(-i\left(\frac{l_{k} \theta}{2}+\psi_{k}(\theta)\right)\right)\right) .
\end{aligned}
$$


Но согласно (1.14) $\exp ( \pm i \nu \theta)=(h(x))^{ \pm 2 \nu}$, и из (1.7) следует, что

$$
\exp \left( \pm i\left(\frac{l_{k} \theta}{2}+\psi_{k}(\theta)\right)\right)=\frac{\sqrt{1-c_{k}} \mathrm{Co}_{l_{k} / 2}(x) \pm \sqrt{1+c_{k}} \operatorname{Si}_{l_{k} / 2}(x)}{\sqrt{1-c_{k} T_{l_{k}}(x)}}
$$

где

$$
\mathrm{Co}_{\nu}(x)=\frac{h^{2 \nu}(x)+h^{-2 \nu}(x)}{2}, \quad \mathrm{Si}_{\nu}(x)=\frac{h^{2 \nu}(x)-h^{-2 \nu}(x)}{2} .
$$

Таким образом, получаем формулу (1.5):

$$
\begin{aligned}
C_{n}(x)= & \frac{1}{2}\left[h^{2 \nu}(x) e^{-i \beta \pi} \prod_{k=1}^{m}\left(\sqrt{1-c_{k}} \mathrm{Co}_{l_{k} / 2}(x)+\sqrt{1+c_{k}} \operatorname{Si}_{l_{k} / 2}(x)\right)\right. \\
& \left.+h^{-2 \nu}(x) e^{i \beta \pi} \prod_{k=1}^{m}\left(\sqrt{1-c_{k}} \mathrm{Co}_{l_{k} / 2}(x)-\sqrt{1+c_{k}} \operatorname{Si}_{l_{k} / 2}(x)\right)\right] \\
& \times(1+x)^{-\alpha}(1-x)^{-\beta} \\
= & \frac{\cos (N \theta-\beta \pi+\psi(\theta))}{w(x, \alpha, \beta, C)}=\frac{\cos (N \theta-\beta \pi+\psi(\theta))}{(1+x)^{\alpha}(1-x)^{\beta}} \sqrt{S_{l}(x)} .
\end{aligned}
$$

График $C_{n}(x)$ будет “ужом" [18] на отрезке $[-1,1]$ в коридоре, ограниченном функциями $\pm 1 / w(x)$. Заметим, что второе слагаемое в квадратных скобках (1.17) обрашается в нуль в корнях $S_{l}(x)$.

Если учесть формулы (1.14) для $h(x)$ и $h^{-1}(x),(1.16)$ для $\mathrm{Co}_{\nu}(x)$ и $\mathrm{Si}_{\nu}(x)$, то мы убедимся, что $C_{n}(x)$ есть функция вида $\sum_{j+k=2 n} A_{j k}(\sqrt{x+1})^{j}(\sqrt{x-1})^{k}$, в которой присутствуют только четные значения $j$ и $k$, т.е. $C_{n}(x)$ есть многочлен степени $n$. Коэффициент $\widetilde{A}_{n}$ при $x^{n}$ у него вычисляется из предельного соотношения $\widetilde{A}_{n}=\lim _{x \rightarrow+\infty} C_{n}(x) / x^{n}:$ при $\nu>0$

$$
\widetilde{A}_{n}=2^{N-1} 2^{-m} \prod_{k=1}^{m}\left(\sqrt{1-c_{k}}+\sqrt{1+c_{k}}\right), \quad \frac{\widetilde{A}_{n+1}}{\widetilde{A}_{n}}=2,
$$

т.е.

$$
\widetilde{A}_{n}^{2}=2^{2(N-1)} B_{m}(C), \quad B_{m}(C)=2^{-m} \prod_{k=1}^{m}\left(1+\sqrt{1-c_{k}^{2}}\right) .
$$

Пусть

$$
\Phi(\theta)=N \theta-\beta \pi+\psi(\theta)
$$

- полная фазовая функция. Тогда из способа вывода формулы (1.17) следует

Лемма 1. Пусть $\Phi_{k}(\theta), k=1,2,-$ полные фазовые функции для весовых функций вида (1.1):

$$
w_{k}(x)=w_{k}\left(x, \alpha_{k}, \beta_{k}, C_{k}\right)=\frac{(1+x)^{\alpha_{k}}(1-x)^{\beta_{k}}}{\sqrt{S_{l_{k}}(x)}},
$$

где $S_{l_{k}}(x)$ - положительные на $[-1,1]$ многочлены вида (1.4) степени $l_{k}$ $\alpha=\alpha_{1}+\alpha_{2}=0, \frac{1}{2}, \beta=\beta_{1}+\beta_{2}=0, \frac{1}{2}$. Тогда функиия $w(x)=w_{1}(x) w_{2}(x)$ будет nри $S_{l}(x)=S_{l_{1}}(x) S_{l_{2}}(x)$ весовой функиией вида (1.1), для которой полной фазовой функиией при $2 N>l_{1}+l_{2}$ будет функиия $\Phi(\theta)=\Phi_{1}(\theta)+\Phi_{2}(\theta)-n \theta$. 
ПримеР 1. На рис. 1-3 приведены графики² : на рис. 1 - весовой функции

$$
w(x)=\frac{1}{\sqrt{1-T_{5}(x) / 1.0006}},
$$

имеюшей три острых пика, на рис. 2 - фазовой функции $\psi(\arccos x)$, на рис. 3 ЧМБС-многочлена $C_{40}(x, 0,0, C)$.

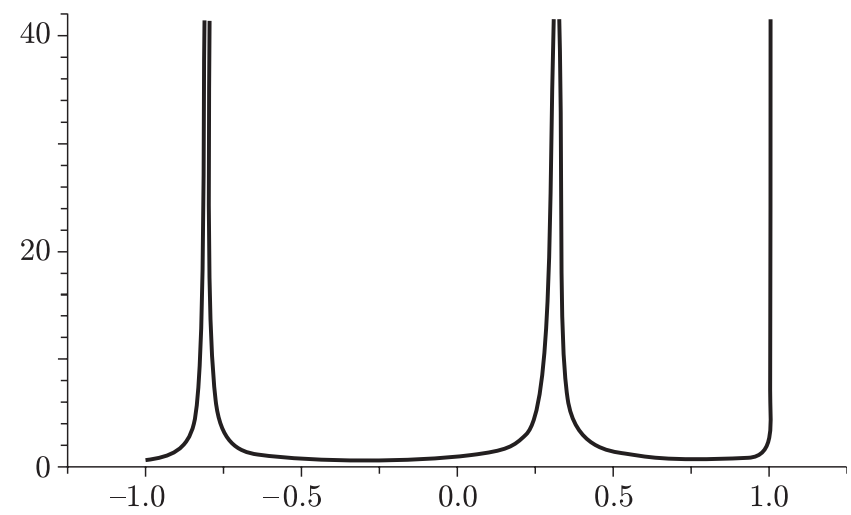

Рис. 1. Весовая функция $w(x)=1 / \sqrt{1-T_{5}(x) / 1.0006}(1.21)$

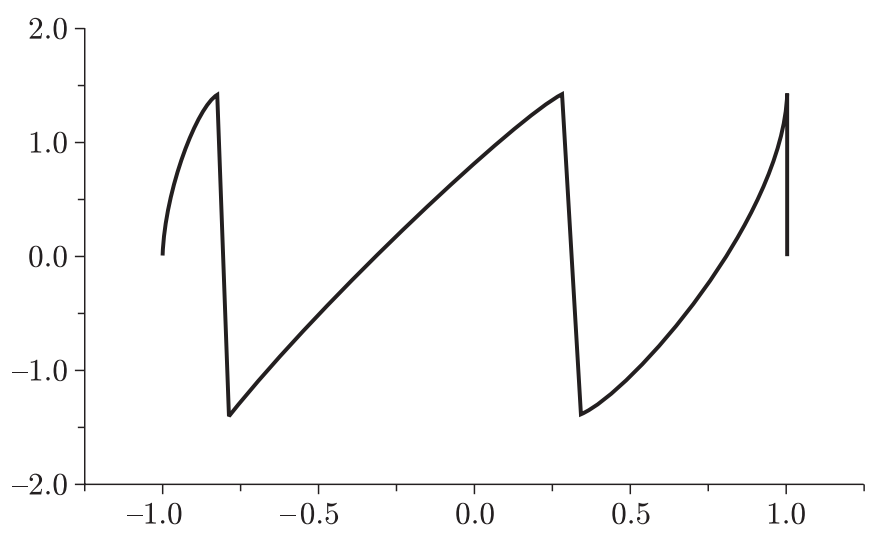

Рис. 2. Фазовая функция $\psi(\arccos x)$ для $(1.21)$

Дадим следуюшие видоизменения формул (1.7), (1.17):

$$
\begin{aligned}
& \exp \left(i \psi_{k}(\theta)\right) \\
& \quad=\frac{\left(\sqrt{1-c_{k}} \cos \left(l_{k} \theta / 2\right)+i \sqrt{1+c_{k}} \sin \left(l_{k} \theta / 2\right)\right)\left(\cos \left(l_{k} \theta / 2\right)-i \sin \left(l_{k} \theta / 2\right)\right)}{\sqrt{1-c_{k} \cos \left(l_{k} \theta\right)}} \\
& \quad=\frac{r_{k}\left(\exp \left(-i l_{k} \theta\right)\right)}{\sqrt{1-c_{k} \cos \left(l_{k} \theta\right)}},
\end{aligned}
$$

\footnotetext{
${ }^{2}$ Графики функций на рисунках в статье получены с помощью программы KLM, составленной В. Д. Коваленко, В. И. Лебедевьм, А. А. Медовиковым.
} 


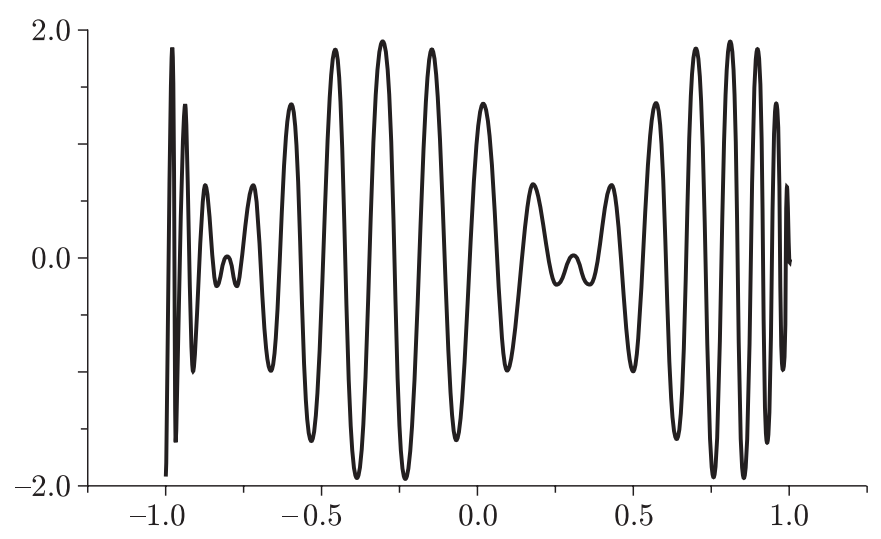

Рис. 3. ЧМБС-многочлен $C_{40}(x, 0,0, C)$ для $(1.21)$

где

$$
r_{k}(z)=\frac{\left(\sqrt{1-c_{k}}+\sqrt{1+c_{k}}\right)-\left(\sqrt{1+c_{k}}-\sqrt{1-c_{k}}\right) z}{2}
$$

причем

$$
r_{k}\left(\exp \left(i l_{k} \theta\right)\right) r_{k}\left(\exp \left(-i l_{k} \theta\right)\right)=1-c_{k} \cos \left(l_{k} \theta\right)
$$

Тогда формула для $C_{n}(x, \alpha, \beta, C)$ примет вид:

$$
\begin{aligned}
C_{n}(x, \alpha, \beta, C)= & \frac{1}{2}\left[\exp (i N \theta) \prod_{k=1}^{m} r_{k}\left(\exp \left(-i l_{k} \theta\right)\right)\right. \\
& \left.+\exp (-i N \theta) \prod_{k=1}^{m} r_{k}\left(\exp \left(i l_{k} \theta\right)\right)\right](1+x)^{-\alpha}(1-x)^{-\beta}
\end{aligned}
$$

Покажем,что множество ЧМБС-многочленов каждого рода с точностью до множителя инвариантно относительно некоторых линейных интегральных преобразований над параметрами $C$. Разберем простейший случай. Пусть $-1<a_{1} \leqslant c_{1} \leqslant$ $b_{1}<1, j_{1}\left(c_{1}\right)>0$ - непрерьвная на $\left[a_{1}, b_{1}\right]$ функция, $d \sigma_{1}\left(c_{1}\right)$ - распределение на $\left[a_{1}, b_{1}\right]$ в интеграле Стилтьеса

$$
J_{1}(f)=\int_{a_{1}}^{b_{1}} f\left(c_{1}\right) j_{1}\left(c_{1}\right) d \sigma_{1}\left(c_{1}\right)
$$

Тогда $J_{1}\left(C_{n}(x, \alpha, \beta, C)\right)$ с точностью до множителя будет ЧМБС-многочленом с весовой функцией $(1.1),(1.4)$, в которой параметр $c_{1}$ заменен на новый $\widetilde{c}_{1}$. Пусть $p=J_{1}\left(\sqrt{1-c_{k}}+\sqrt{1+c_{k}}\right), q=J_{1}\left(\sqrt{1-c_{k}}-\sqrt{1+c_{k}}\right)$, тогда из (1.25) следует, что после интегрирования в $(1.25)$ множители $r_{1}\left(\exp \left( \pm i l_{k} \theta\right)\right)$ заменятся на $J_{1}\left(r_{1}\left(\exp \left( \pm i l_{k} \theta\right)\right)\right)=\left(p-q \exp \left( \pm i l_{k} \theta\right)\right) / 2$, т.е. $\widetilde{c}_{1}=2 p q /\left(p^{2}+q^{2}\right)$. Аналогичные преобразования возможны и по другим параметрам $C$. 


\section{§2. Свойства ЧМБС-многочленов}

Вычислим значения $C_{n}( \pm 1, \alpha, \beta, C)$ :

$$
\begin{aligned}
C_{n}(1,0,0, C)=\sqrt{S_{l}(1)}, \quad C_{n}(-1,0,0, C)=(-1)^{n} \sqrt{S_{l}(-1)} \\
C_{n}\left(1, \frac{1}{2}, 0, C\right)=\sqrt{\frac{S_{l}(1)}{2}} \\
C_{n}\left(-1, \frac{1}{2}, 0, C\right)=(-1)^{n} \sqrt{2 S_{l}(-1)}\left(n+\frac{1}{2}+\psi^{\prime}(\pi)\right) \\
C_{n}\left(1,0, \frac{1}{2}, C\right)=\sqrt{2 S_{l}(1)}\left(n+\frac{1}{2}+\psi^{\prime}(0)\right) \\
C_{n}\left(-1,0, \frac{1}{2}, C\right)=(-1)^{n} \sqrt{\frac{S_{l}(-1)}{2}} \\
C_{n}\left(1, \frac{1}{2}, \frac{1}{2}, C\right)=\sqrt{S_{l}(1)}\left(n+1+\psi^{\prime}(0)\right), \\
C_{n}\left(-1, \frac{1}{2}, \frac{1}{2}, C\right)=(-1)^{n} \sqrt{S_{l}(-1)}\left(n+1+\psi^{\prime}(\pi)\right) .
\end{aligned}
$$

Справедливы равенства

$$
\begin{aligned}
C_{n}^{2}(x, 0,0, C)+\left(1-x^{2}\right) C_{n-1}^{2}\left(x, \frac{1}{2}, \frac{1}{2}, C\right) & =S_{l}(x), \\
(1+x) C_{n}^{2}\left(x, \frac{1}{2}, 0, C\right)+(1-x) C_{n}^{2}\left(x, 0, \frac{1}{2}, C\right) & =S_{l}(x),
\end{aligned}
$$

которые определяют конструктивный алгоритм построения формул в теореме Маркова-Лукача [18] для представления положительного на $[-1,1]$ многочлена $S_{l}(x)$. Из этих формул и (1.5) следует, что точками локального максимума на $[-1,1] \mathrm{sB}-$ ляются $x= \pm 1$ для $\left|C_{n}(x, 0,0, C)\right|, x=1$ для $(1+x)^{1 / 2}\left|C_{n}\left(x, \frac{1}{2}, 0, C\right)\right|, x=-1$ для $(1-x)^{1 / 2}\left|C_{n}\left(x, 0, \frac{1}{2}, 0, C\right)\right|$. Справедлива

Теорема 1. Многочлены $\widetilde{A}_{n}^{-1} C_{n}(x, \alpha, \beta, C)$ с единичным коэффициентом при $x^{n}$ обладают обобщенным альтернансом из $n+1$ точки и являются экстремальными с весом (1.1), (1.4) многочленами (решением задачи I).

Покажем, что произведение $C_{n}(x, \alpha, \beta, C) w(x, \alpha, \beta, C)$ имеет чебышёвский альтернанс по крайней мере из $n+1$ точки [19]. Для этого рассмотрим непрерывную полную фазовую функцию $\Phi(\theta)$ (1.20). Вследствие условий (1.12) функция $\Phi(\theta)$ изменяется на отрезке $[0, \pi]$ от $-\beta \pi$ до $(n+\alpha) \pi$. Следовательно, для каждого $j=\overline{0, n}$ существует $\widetilde{\theta}_{j} \in[0, \pi]$ такая, что $\Phi\left(\widetilde{\theta}_{j}\right)=j \pi, j=\overline{0, n}$. Это и будут точки альтернанса, так как в них рассматриваемые функции принимают с переменой знака одинаковые по модулю максимальные значения. Если бы существовал другой многочлен $n$-й степени рассматриваемого класса $P_{n}(x)$ с меньшим взвешенньм отклонением от нуля, то разность $\widetilde{A}_{n}^{-1} C_{n}(x, \alpha, \beta, C)-P_{n}(x)$, будучи многочленом степени $n-1$, поочередно в $n+1$ точке $\widetilde{\theta}_{j}$ меняла бы знак, что невозможно. 
Справедливо

СлЕДСТВИЕ 1. Для многочлена степени п вида

$$
P_{n}(x)=P_{n-1}(x)+a_{n} C_{n}(x, \alpha, \beta, C), \quad a_{n} \neq 0,
$$

наименее отклоняющимся от него многочленом с весом (1.1), (1.4) степени $n-1$ будет многочлен $P_{n-1}(x)$.

Для приложений полезна (см. §5) следуюшая нормировка ЧМБС-многочленов в задаче I: пусть $\delta>1$, найти

$$
\begin{aligned}
e_{n} & =\min _{q_{n}(\delta)=1} \max _{x \in[-1,1]}\left|q_{n}(x) w(x)\right|, \\
p_{n}(x) & =\arg \min _{q_{n}(\delta)=1} \max _{x \in[-1,1]}\left|q_{n}(x) w(x)\right| .
\end{aligned}
$$

Покажем, что решением этой задачи I будет

$$
p_{n}(x)=\widetilde{C}_{n}(x, \alpha, \beta, C)=\frac{C_{n}(x)}{C_{n}(\delta)}, \quad e_{n}=\frac{1}{\left|C_{n}(\delta)\right|} .
$$

Теорема 2. Многочлен $\widetilde{C}_{n}(x, \alpha, \beta, C)$ с единичныц значением при $x=\delta>1$ является әкстремальныцм с весом (1.1), (1.4) многочленом (решением задачu I).

Произведение $\widetilde{C}_{n}(x, \alpha, \beta, C) w(x, \alpha, \beta, C)$ имеет чебышёвский альтернанс по крайней мере из $n+1$ точки $\widetilde{\theta}_{j} \in[0, \pi]$. Если бы существовал другой многочлен $n$-й степени рассматриваемого класса $P_{n}(x)$ с меньшим взвешенным отклонением от нуля, то разность $\widetilde{C}_{n}(x, \alpha, \beta, C)-P_{n}(x)$, будучи многочленом степени $n$, имела бы $n$ нулей между точками $\widetilde{\theta}_{j}$ и еще один нуль при $x=\delta>1$, что невозможно. Формула для $e_{n}$ в (2.5) следует из того, что для $w(x)$ вида $(1.1),(1.4)$ $\max _{x \in[-1,1]}\left|C_{n}(x) w(x)\right|=1$.

Из теоремы 2 вытекает

СлЕДСТвИЕ 2. Среди всех многочленов $Q_{n}(x)$-й степени, подчиненных условию $\max _{x \in[-1,1]}\left|Q_{n}(x) w(x)\right| \leqslant \eta>0$, наибольшее по модулю значение в точке $\delta>1$ принимают многочлены $\widetilde{P}_{n}(x)= \pm \eta C_{n}(x)$.

Следствие легко устанавливается доказательством от противного.

Вычислим $C_{n}(\delta)$ в $(2.5)$ при $\alpha=\beta=0$. Пусть $\tau=\left(\delta+\sqrt{\delta^{2}-1}\right)^{-1}$, тогда $h(\delta)=\tau^{-1 / 2}, \mathrm{Co}_{\mu}(\delta)=\left(\tau^{-\mu}+\tau^{\mu}\right) / 2, \operatorname{Si}_{\mu}(\delta)=\left(\tau^{-\mu}-\tau^{\mu}\right) / 2$ и

$$
\begin{aligned}
C_{n}(\delta)= & \frac{\tau^{-n}}{2^{m+1}}\left[\prod_{k=1}^{m}\left(\sqrt{1-c_{k}}\left(1+\tau^{l_{k}}\right)+\sqrt{1+c_{k}}\left(1-\tau^{l_{k}}\right)\right)\right. \\
& \left.+\tau^{2 n-l} \prod_{k=1}^{m}\left(\sqrt{1-c_{k}}\left(1+\tau^{l_{k}}\right)-\sqrt{1+c_{k}}\left(1-\tau^{l_{k}}\right)\right)\right],
\end{aligned}
$$

а при $1 \leqslant l \leqslant 2 n, c_{k}=\delta^{-1}, l_{k}=1$,

$$
S_{l}(x)=\left(1-\delta^{-1} x\right)^{l}, \quad \psi(\theta)=l \operatorname{arctg} \frac{\sin \theta}{\tau^{-1}-\cos \theta}
$$


имеем: $\sqrt{1-\delta^{-1}} \operatorname{Co}_{1 / 2}(\delta)=\sqrt{1+\delta^{-1}} \operatorname{Si}_{1 / 2}(\delta)=\sqrt{\left(\delta^{2}-1\right) /(2 \delta)}$, т.е.

$$
\begin{aligned}
C_{n}(\delta) & =\frac{\tau^{-n+l / 2}}{2}\left(\frac{2\left(\delta^{2}-1\right)}{\delta}\right)^{l / 2}, \\
e_{l, n} & =e_{n}=2 \tau^{n-l / 2}\left(\frac{\delta}{\left(2\left(\delta^{2}-1\right)\right.}\right)^{l / 2} .
\end{aligned}
$$

ПРимеР 2. Пусть $S_{l}(x)$ из (2.6), $l=2, \delta=1.01$. На рис. 4-6 приведены графики: на рис. 4 - весовой функции $w(x)$, на рис. 5 - фазовой функции $\psi(\arccos x)$, на рис. 6 - ЧМБС-многочлена $C_{40}(x, 0,0, C)$.

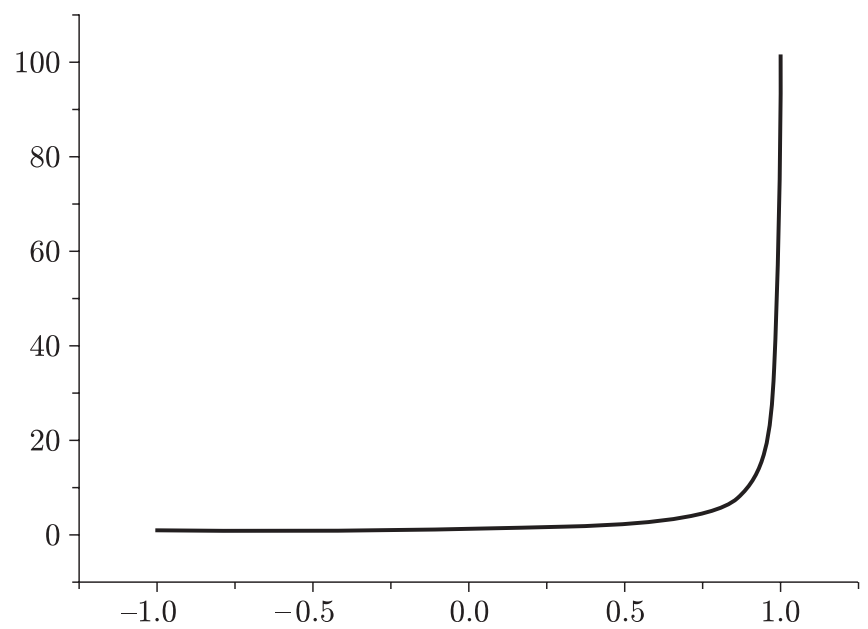

Рис. 4. Весовая функция $w(x)$ для (2.6)

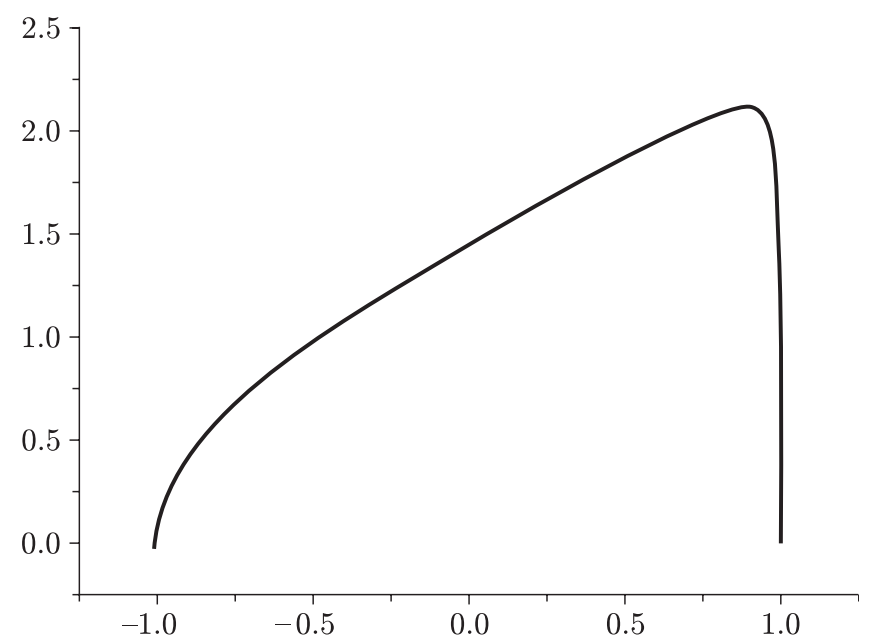

Рис. 5. Фазовая функция $\psi(\arccos x)(2.6)$ 


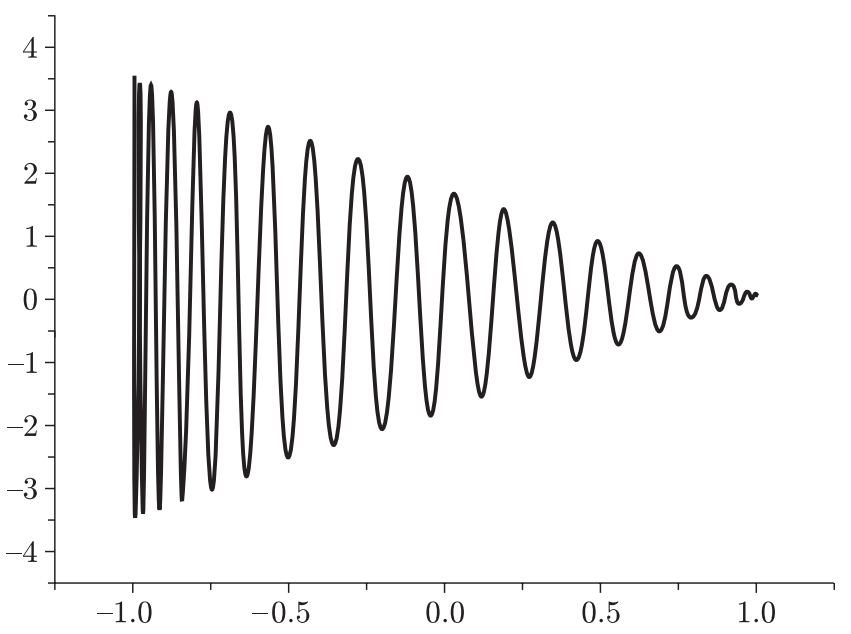

Рис. 6. ЧМБС-многочлен $C_{40}(x, 0,0, C)$ для $(2.6)$

Корни $x_{i}$ многочлена $C_{n}(x)$ удовлетворяют уравнениям

$$
x_{i}=\cos \theta_{i}, \quad \theta_{i}=\theta_{i}^{0}-\frac{\psi\left(\theta_{i}\right)}{N}, \quad i=\overline{1, n}
$$

Сушествование решений уравнений (2.7) следует из того, что функция $\Phi(\theta)$ для каждого $i=\overline{1, n}$ обязательно примет значение $\left(i-\frac{1}{2}\right) \pi$. При

$$
\max _{\theta \in[0, \pi]} \frac{\left|\psi^{\prime}\right|}{N}<1
$$

для каждого $i$ решение будет единственньм, и $\theta_{i}$ могут быть найдены итерационным методом сжимающих отображений

$$
\theta_{i}^{j+1}=\theta_{i}^{0}-\frac{\psi\left(\theta_{i}^{j}\right)}{N}, \quad i=\overline{1, n}, \quad j=0,1, \ldots,
$$

или методом Ньютона (при его сходимости)

$$
\theta_{i}^{j+1}=\theta_{i}^{j}-\frac{N\left(\theta_{i}^{j}-\theta_{i}^{0}\right)+\psi\left(\theta_{i}^{j}\right)}{N+\psi^{\prime}\left(\theta_{i}^{j}\right)}, \quad i=\overline{1, n}, \quad j=0,1, \ldots,
$$

начиная с начального приближения $\theta_{i}^{0}=\left(i+\beta-\frac{1}{2}\right) \pi / N$. При больших $n$ корни $x_{i}$ близки к корням соответствующих классических чебьшёвских многочленов $\widetilde{x}_{i}=\cos \theta_{i}^{0}$. Делая одну итерацию по методу Ньютона, получаем асимптотические приближения для $\theta_{i}: \theta_{i} \approx \theta_{i}^{1}=\theta_{i}^{0}-b_{i}, b_{i}=\psi\left(\theta_{i}^{0}\right) /\left(N+\psi^{\prime}\left(\theta_{i}^{0}\right)\right)$, т.е.

$$
x_{i} \approx \widetilde{x}_{i} \cos b_{i}+\left(1-\widetilde{x}_{i}^{2}\right)^{1 / 2} \sin b_{i}, \quad i=\overline{1, n} .
$$

Пусть

$$
p(x, C)=p(x, \alpha, \beta, C)=\frac{(1+x)^{2 \alpha-1 / 2}(1-x)^{2 \beta-1 / 2}}{S_{l}(x)},
$$


тогда

$$
\begin{aligned}
(1-x) p(x, \alpha, 0, C) & =p\left(x, \alpha, \frac{1}{2}, C\right), \quad(1+x) p(x, 0, \beta, C)=p\left(x, \frac{1}{2}, \beta, C\right) \\
& \left(1-x^{2}\right) p(x, 0,0, C)=p\left(x, \frac{1}{2}, \frac{1}{2}, C\right)
\end{aligned}
$$

ЧМБС-многочлены при $N \geqslant \frac{l}{2}$ ортогональны в следуюшем смысле [6], [9]:

$$
\int_{-1}^{1} x^{k} C_{n}(x) \frac{(1+x)^{2 \alpha-1 / 2}(1-x)^{2 \beta-1 / 2}}{S_{l}(x)} d x=0, \quad k=\overline{0, n-1}
$$

или, используя многочлены (0.5) степени $k$, получаем эквивалентные равенства

$$
I=\int_{0}^{\pi} \frac{\cos ((k+\alpha+\beta) \theta-\beta \pi) \cos (N \theta-\beta \pi+\psi(\theta))}{\sqrt{\prod_{k=1}^{m}\left(1-c_{k} \cos \left(l_{k} \theta\right)\right)}} d \theta=0, \quad k=\overline{0, n-1} .
$$

Докажем единьм методом ортогональность для общего случая. Имеем

$$
I=\frac{(-1)^{2 \beta} I_{1}+I_{2}}{2}
$$

где

$$
I_{1}=\int_{0}^{\pi} \frac{\cos \left(n_{1} \theta+\psi(\theta)\right)}{\sqrt{\prod_{k=1}^{m}\left(1-c_{k} \cos \left(l_{k} \theta\right)\right)}} d \theta, \quad I_{2}=\int_{0}^{\pi} \frac{\cos \left(n_{2} \theta+\psi(\theta)\right)}{\sqrt{\prod_{k=1}^{m}\left(1-c_{k} \cos \left(l_{k} \theta\right)\right)}} d \theta,
$$

а $n_{1}=n+k+2(\alpha+\beta), n_{2}=n-k$. Покажем, что $I_{1}=I_{2}=0$. В самом деле, для $j=1,2, k=\overline{0, n-1}$

$$
I_{j}=\frac{1}{2} \int_{-\pi}^{\pi} \frac{\cos \left(n_{j} \theta+\psi(\theta)\right)}{\sqrt{\prod_{k=1}^{m}\left(1-c_{k} \cos \left(l_{k} \theta\right)\right)}} d \theta=\frac{1}{2} \operatorname{Re} \int_{-\pi}^{\pi} \frac{\exp \left(i\left(n_{j} \theta+\psi(\theta)\right)\right)}{\sqrt{\prod_{k=1}^{m}\left(1-c_{k} \cos \left(l_{k} \theta\right)\right)}} d \theta .
$$

Используя соотношения (1.22)-(1.24) и делая затем замену переменной интегрирования $z=\exp (i \theta)$, получаем

$$
I_{j}=\frac{1}{2} \operatorname{Re} \int_{-\pi}^{\pi} \exp \left(i n_{j} \theta\right) \prod_{k=1}^{m} \frac{r_{k}\left(\exp \left(-i l_{k} \theta\right)\right)}{1-c_{k} \cos \left(l_{k} \theta\right)} d \theta=\frac{1}{2 i} \operatorname{Re} \int_{|z|=1} \frac{z^{n_{j}-1}}{R_{l}(z)} d z
$$

где $R_{l}(z)=\prod_{k=1}^{m} r_{k}\left(z^{l_{k}}\right)$ - многочлен, имеющий корни вне окружности $|z|=1$. Следовательно, $I_{j}=0, j=1,2$, т.е. $I=0$.

Умноженные на $\sqrt{2 / \pi}$ ЧМБС-многочлены будут ортонормированньми с весом $p(x, \alpha, \beta, C)$ при $N>\frac{l}{2}$ :

$$
\int_{-1}^{1} C_{n}^{2}(x) w^{2}(x, \alpha, \beta, C)\left(1-x^{2}\right)^{-1 / 2} d x=\frac{\pi}{2}
$$


В самом деле, согласно (1.5)

$$
\begin{aligned}
\int_{-1}^{1} & C_{n}^{2}(x) w^{2}(x, \alpha, \beta, C)\left(1-x^{2}\right)^{-1 / 2} d x=\int_{0}^{\pi} \cos ^{2}(N \theta-\beta \pi+\psi(\theta)) d \theta \\
= & \frac{1}{2}\left(\pi+\int_{0}^{\pi} \cos (2(N \theta-\beta \pi+\psi(\theta))) d \theta\right)
\end{aligned}
$$

но согласно лемме 1 и (2.12)

$$
\int_{0}^{\pi} \cos (2(N \theta-\beta \pi+\psi(\theta))) d \theta=\int_{-1}^{1} C_{2 N}(x, 0,0, \bar{C}) \frac{\left(1-x^{2}\right)^{-1 / 2}}{S_{l}^{2}(x)} d x=0
$$

где $C_{2 N}(x, 0,0, \bar{C})$ - ЧМБС-многочлен для $w(x)=1 / S_{l}(x)$. Нам неизвестны аналитические формулы для ЧМБС-многочленов низших степеней. Однако стандартным процессом Сонина-Шмидта мы построим ортонормированную условиями (2.12), (2.13) систему многочленов. При этом интегралы в алгоритме ортогонализации можно вычислять точно по квадратурным формулам типа Гаусса (см. $\S 4)$. Эта система для $N \geqslant \frac{l}{2}$ совпадет с рассмотренными многочленами. Для нормированных многочленов справедлива формула Кристоффеля-Дарбу [9]:

$$
\begin{aligned}
& D_{n}(x, y, \alpha, \beta)=\sum_{i=0}^{n} C_{i}(x, \alpha, \beta, C) C_{i}(y, \alpha, \beta, C) \\
& \quad=\frac{C_{n+1}(x, \alpha, \beta, C) C_{n}(y, \alpha, \beta, C)-C_{n+1}(y, \alpha, \beta, C) C_{n}(x, \alpha, \beta, C)}{2(x-y)}
\end{aligned}
$$

где

$$
C_{0}^{2}(x, \alpha, \beta, C)=\frac{\pi}{2}\left(\int_{-1}^{1} p(x, \alpha, \beta, C) d x\right)^{-1}
$$

Многочлены $D_{n}(x, y)$ образуют ортогональную с весом $(x-y) p(x, \alpha, \beta, C)$ систему многочленов. Нам понадобятся следующие равенства:

$$
\int_{-1}^{1} D_{n}(x, y, \alpha, \beta) p(x, \alpha, \beta, C) d x=\frac{\pi}{2}
$$

и при $\beta=0$

$$
\begin{aligned}
& D_{n}(x, 1, \alpha, 0)=\frac{\sin (n+\alpha+1 / 2+\psi(\theta))}{2 w(x, \alpha, 0, C) w(1, \alpha, 0, C) \sin (\theta / 2)}=\frac{C_{n}(x, \alpha, 1 / 2, C)}{\sqrt{2} w(1, \alpha, 0, C)} \\
& D_{n}(1,1, \alpha, 0)=\left(n+\alpha+\frac{1}{2}+\psi^{\prime}(0)\right) S_{l}(1) 2^{-2 \alpha}
\end{aligned}
$$

При $n \geqslant n_{0}$, где $n_{0}=\min \left(k: k \geqslant \frac{l}{2}+1\right)$, ЧМБС-многочлены удовлетворяют чебьшёвским трехчленньм рекуррентньм соотношениям: из (1.5) получаем

$$
C_{n+1}(x)=2 x C_{n}(x)-C_{n-1}(x)
$$


При $n<n_{0} C_{n}(x)$ и коэффициенты трехчленных рекуррентных соотношений последовательно при $n=n_{0}-1, \ldots, 1$ определяются алгоритмом Евклида - делением $C_{n+2}(x)$ на $C_{n+1}(x)$ с остатком, считая каждый многочлен $C_{n}(x)$ некоторым способом нормированными, а, например, $C_{0}(x) \equiv 1$. При $l=1,2$ систему уравнений (2.19) достаточно дополнить одним уравнением при известных $C_{0}(x)$ и $C_{1}(x), C_{2}(x)$, вычисленных из (1.17):

$$
C_{2}(x)=\left(a_{2} x+b_{2}\right) C_{1}(x)-d_{2} C_{0}(x) .
$$

Выражая $C_{k}(x)$ через $\widetilde{C}_{k}(x), k=n-1, n, n+1$, и подставляя эти значения в (1.17), получаем

$$
\widetilde{C}_{n+1}(x)=2 \frac{C_{n}(\delta)}{C_{n+1}(\delta)} x \widetilde{C}_{n}(x)-\frac{C_{n-1}(\delta)}{C_{n+1}(\delta)} \widetilde{C}_{n-1}(x) .
$$

Многочлены, удовлетворяющие трехчленньм рекуррентным соотношениям, коэффициенты в которых начиная с некоторого номера $n_{0}$ становятся периодичными с периодом $s$, были изучены в работах Геронимуса [20]-[22]; для этих многочленов также сушествует тригонометрическая форма их представления [23].

Сделаем четыре замечания.

1) Пусть $R_{M}(x)$ - многочлен $M$-й степени, все корни которого лежат на $[-1,1]$, а в (1.1) $w(x)=w(x, \alpha, \beta, C) /\left|R_{M}(x)\right|$, тогда [7] экстремальньм многочленом степени $n+M$ - решением задачи I - будет многочлен

$$
\widetilde{P}_{n+M}(x)=R_{M}(x) C_{n}(x),
$$

где $C_{n}(x)$ - многочлен (1.17).

2) Пусть для $x \in[-1,1]$

$$
\begin{gathered}
S(x)=S(x, c, d)=\left(1+c(1-x)+d(1-x)^{2}\right)>0, \\
w(x, c, d, n)=S(x, c, d)^{-n / 2}, \quad w(1, c, d, n)=1 .
\end{gathered}
$$

Оказывается, что для этого случая $C_{n}(x)$ и формулы для его корней имеют простой вид. Пусть

$$
z(x)=\frac{g(x)}{\sqrt{S(x)}}, \quad g(x)=\frac{x+1+\sqrt{S(-1)}(x-1)}{2},
$$

- преобразование, отображающее взаимно однозначно отрезок $[-1,1]$ на себя, $z(1)=1, z(-1)=-1$, тогда

$$
C_{n}(x)=\bar{C}_{n}(x, c, d)=T_{n}(z(x)) \sqrt{S^{n}(x)} .
$$

Используя формулу для $T_{n}(z)$, получаем

$$
\bar{C}_{n}(x, c, d)=\frac{1}{2}\left(\left(g(x)+\sqrt{g^{2}(x)-S(x)}\right)^{n}+\left(g(x)-\sqrt{g^{2}(x)-S(x)}\right)^{n}\right) .
$$


Корни $x_{i} \in[-1,1]$ этого многочлена $C_{n}(x)$ определяются через корни $\beta_{i}=$ $\cos \theta_{i}^{0}$ многочлена $T_{n}(x)$ из уравнений $z\left(x_{i}\right)=\beta_{i}, i=1, \ldots, n$, которые преобразуются в квадратные уравнения $g^{2}\left(x_{i}\right)=\beta_{i}^{2} S\left(x_{i}\right)$. Искомьм корнем для каждого $i$ будет максимальный корень при $\beta_{i} \geqslant 0$ и минимальный корень при $\beta_{i}<0$ квадратного уравнения.

Пусть $S(-1)=1+2 c+4 d>1, c>0$, тогда $\delta_{1}=g(\delta)=\delta+(\sqrt{S(-1)}-1) \times$ $(\delta-1) / 2>\delta, \tau_{1}=\left(\delta_{1}+\sqrt{\delta_{1}^{2}-S(\delta)}\right)^{-1}=\left(\delta_{1}+\sqrt{\left(\delta^{2}-1\right)(1+3 c / 2+2 d)}\right)^{-1}<1$. Величина $e_{n}(2.3)$ в этом случае при $S(\delta) \neq 0$ равна

$$
e_{n}=e_{n}(c, d)=\frac{1}{\left|T_{n}(z(\delta)) \sqrt{S^{n}(\delta)}\right|}
$$

или из $(2.25)$

$$
e_{n}(c, d)=\frac{2 \tau_{1}^{n}}{1+\left(S(\delta) \tau_{1}^{2}\right)^{n}}
$$

А при $S(\delta)=0$ :

$$
e_{n}(c, d)=2^{1-n} \tau_{1}^{n}
$$

Пусть

$$
v(x)=\frac{R(x)}{R(1)}, \quad R(x)=\prod_{k=1}^{m}\left(x+a_{k}\right), \quad a_{k}>1 .
$$

Рассмотрим задачу I $(2.3),(2.4)$ при $w(x)=v(x)$ : при $\delta>1$ найти

$$
p_{n}(x)=\arg \min _{q_{n}(\delta)=1} \sup _{x \in[-1,1]}\left|q_{n}(x) v(x)\right|, \quad p_{n}(\delta)=1 .
$$

Аналитическое ее решение и величина $\widetilde{e}_{n}=e_{n}$ неизвестны, хотя известны достаточно точные асимптотические формулы для ее параметров [13]. С помощью функции $w(x, c, d, n)$ дадим двусторонние оценки для $\widetilde{e}_{n}$ и многочлены, реализующие эти оценки. Имеем $w(1, c, d, n)=v(1)$ и для $x \in[-1,1]: v(x)>0$, $v^{\prime}(x)>0, v^{\prime \prime}(x) \geqslant 0, v^{\prime}(x) / v(x)=\sum_{i=1}^{m} 1 /\left(x+a_{i}\right)>0, \quad\left(v^{\prime}(x) / v(x)\right)^{\prime}=$ $-\sum_{i=1}^{m} 1 /\left(x+a_{i}\right)^{2}<0,0<v(-1)=\prod_{k=1}^{m}\left(1-2 /\left(1+a_{k}\right)\right)<1$. Пусть $y(x)=(v(x))^{-2 / n}$, тогда для $x \in[-1,1]: \quad y(x)$ - положительная убываюшая функция, $y(1)=1 ; y^{\prime}(x)=-(2 / n) y(x) v^{\prime}(x) / v(x)$ - отрицательная возрастающая функция; $y^{\prime \prime}(x)=\left(4 / n^{2}\right) y(x)\left(v^{\prime}(x) / v(x)\right)^{2}-(2 / n) y(x)\left(v^{\prime}(x) / v(x)\right)^{\prime}$ - положительная убываюшая функция.

Параметр $с$ определим из условия $S^{\prime}(1, c, d, n)=y^{\prime}(1)$, тогда

$$
c=\frac{2}{n} \sum_{i=1}^{m} \frac{1}{1+a_{i}} .
$$

При $d=d_{0}$, где

$$
d_{0}=\frac{c^{2}}{2}+\frac{1}{n} \sum_{i=1}^{m} \frac{1}{\left(1+a_{i}\right)^{2}}
$$


$S^{\prime \prime}\left(1, c, d_{0}, n\right)=y^{\prime \prime}(1)$ и $S\left(x, c, d_{0}, n\right) \leqslant y(x), x \in[-1,1]$. При $d=\widetilde{d}(\widetilde{x})$, где $-1 \leqslant \widetilde{x}<1$,

$$
\widetilde{d}(\widetilde{x})=\frac{(y((\widetilde{x})-1)-1)(1-\widetilde{x})-c / 2}{1-\widetilde{x}},
$$

имеем $S(x, c, \widetilde{d}(\widetilde{x}), n)=y(\widetilde{x})$ и $S(x, c, \widetilde{d}(\widetilde{x}), n) \geqslant y(x)$ при $x \in[\widetilde{x}, 1], S(x, c, \widetilde{d}(\widetilde{x}), n) \leqslant$ $y(x)$ при $x \in[-1, \widetilde{x}]$.

Таким образом,

$$
e_{n}(c, \widetilde{d}(-1)) \leqslant \widetilde{e}_{n} \leqslant e_{n}(c, \bar{d})
$$

Заметим, что

$$
\begin{aligned}
\lim _{n \rightarrow \infty} w\left(x, c, d_{0}, n\right) & =\exp \left((x-1) \sum_{i=1}^{m} \frac{1}{1+a_{i}}-\frac{1}{2}(1-x)^{2} \sum_{i=1}^{m} \frac{1}{\left(1+a_{i}\right)^{2}}\right) \\
\lim _{n \rightarrow \infty} w(x, c, \widetilde{d}(-1), n) & =(v(-1))^{(1-x)^{2} / 4} \exp \left(-\frac{1}{2}\left(1-x^{2}\right) \sum_{i=1}^{m} \frac{1}{1+a_{i}}\right) .
\end{aligned}
$$

Заслуживает внимания приближение с $\widetilde{x}=0$.

ПримеР 3. На рис. 7 приведен график весовой функции $w(x, c, d, 40)$, на рис. 8 график многочлена $\bar{C}_{40}(x, c, d)$ при $c=\frac{3}{20}, d=\frac{1}{8}$.

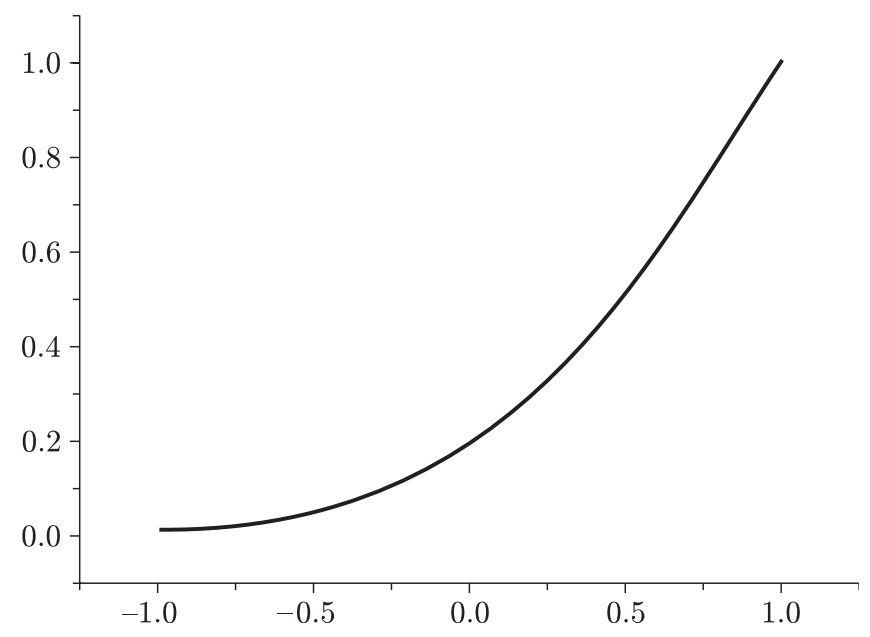

Рис. 7. Весовая функция $w(x, c, d, 40)(2.23)$

Аналогично $M_{n}(x, C)=U_{n}(z(x)) \sqrt{S_{l}(x)}$ будет ЧМБС-многочленом 2-го рода для весовой функции $w(x, C)=\sqrt{\left(1-x^{2}\right) /\left(\left(1-c_{1} x\right)\left(1-c_{2} x\right)\right)^{n+1}}$.

3) Формулы (1.1)-(1.17) позволяют построить явные выражения обобшенных ЧМБС-многочленов для класса весовых функций типа (1.1), (1.4), наименее отклоняющихся от нуля на $L$ специального вида кривых, лежащих в плоскости комплексного переменного $z=x+i y[15],[17],[24]$, [25]. Пусть $Q_{L}(z)$ - многочлен точно $L$-й степени с вешественными коэффициентами, $\rho>0, \Omega_{\rho}=\left\{z:-\rho \leqslant Q_{L}(z) \leqslant \rho\right\}$. 


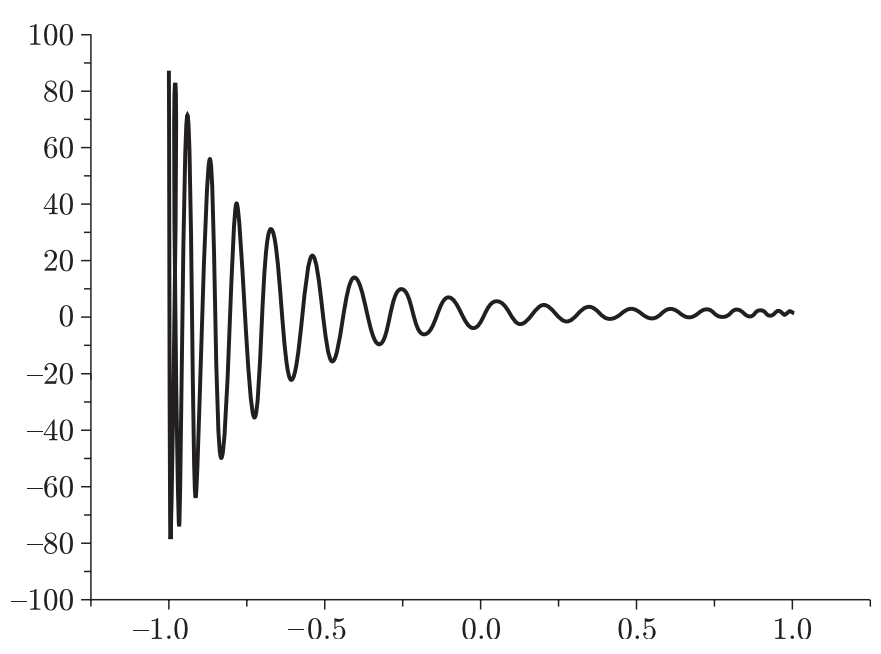

Рис. 8. Многочлен $\bar{C}_{40}(x, c, d)(2.25)$

Множество $\Omega_{\rho}$ состоит из $L$ кривых. Если $Q_{L}(z)$ имеет $L$ действительных однократных корней, тогда сушествует $R>0$ такое, что для $0 \leqslant \rho \leqslant R$ множество $\Omega_{\rho}$ состоит из $L$ изолированных отрезков действительной оси, концами которых служат точки, где $\left|Q_{L}(x)\right|=\rho$. Пусть $x=Q_{L}(z) / \rho$, тогда при $N=L n$ ЧМБС-многочленом, наименее отклоняющимся от нуля на $\Omega_{\rho}$ с весом $(1.1),(1.4)$ при $x=Q_{L}(z) / \rho$, будет многочлен $\bar{P}_{N}(z)=C_{n}\left(Q_{L}(z) / \rho\right)$, где $C_{n}(x)$ и его параметры заданы формулами (1.1)-(1.17). Из (2.19) следует, что при $N>\frac{l}{2}$ для $\bar{P}_{N}(z)$ справедливы уравнения

$$
\bar{P}_{(n+1) L}(z)=\frac{2 Q_{L}(z)}{\rho} \bar{P}_{n L}(z)-\bar{P}_{(n-1) L}(z) .
$$

Корни $\bar{P}_{n L}(z)$ определяются системой уравнений

$$
Q_{L}(z)=\rho x_{k}, \quad k=\overline{1, n} .
$$

4) Интересно изучить свойства последовательностей функций $u_{n}(x)$, являющихся решениями чебьшёвских трехчленных рекуррентных соотношений

$$
u_{n+1}(x)=2 x u_{n}(x)-u_{n-1}(x), \quad n=1,2, \ldots
$$

Общее решение уравнений (2.36) для каждого $x$ представим в двух формах: для любого целого $l>0$

$$
u_{n}(x)=C_{1}(x) T_{n-l+1}(x)+C_{2}(x) U_{n-l}(x),
$$

где $C_{1}(x), C_{2}(x)$ - произвольные функции; а для $-1 \leqslant x=\cos \theta \leqslant 1$ - в тригонометрической форме

$$
u_{n}(x)=\frac{1}{w(x)} \cos ((n-l) \theta+\psi(\theta)),
$$

где $w(x) \neq 0$ в $(-1,1)$ и $\psi(\theta)$ - произвольная ограниченная фазовая функция. 
Пусть $F_{l-1}(x), F_{l}(x)$ являются известными функциями. Определим в $(2.37)$ функции $C_{1}(x), C_{2}(x)$ так, чтобы

$$
u_{l-1}(x)=F_{l-1}(x), \quad u_{l}(x)=F_{l}(x) .
$$

Полагая в (2.37) $n=l-1, l$, учитывая, что $T_{1}(x)=x, T_{0}(x)=1, U_{0}(x)=1$, $U_{-1}(x)=0$, получаем для определения $C_{1}(x), C_{2}(x)$ систему уравнений

$$
C_{1}(x)=F_{l-1}(x), \quad C_{1}(x) x+C_{2}(x)=F_{l}(x),
$$

T.e.

$$
C_{1}(x)=F_{l-1}(x), \quad C_{2}(x)=F_{l}(x)-x F_{l-1}(x) .
$$

Тогда при $n \geqslant l-1$ функция

$$
u_{n}(x)=F_{l-1}(x) T_{n-l+1}(x)+\left(F_{l}(x)-x F_{l-1}(x)\right) U_{n-l}(x)
$$

является искомым решением (2.36). Приведем это решение к тригонометрической форме (2.38). Если обозначить

$$
\begin{aligned}
& g(x)=\frac{x F_{l-1}(x)-F_{l}(x)}{\left(1-x^{2}\right)^{1 / 2}}, \\
& w(x)=\frac{1}{\sqrt{F_{l-1}^{2}(x)+g^{2}(x)}}
\end{aligned}
$$

и задать фазовую функцию $\psi(\theta)$ равенствами [6], [7]:

$$
\begin{aligned}
& \cos ((l-1) \theta+\psi(\theta))=\frac{F_{l-1}(\cos \theta)}{w(\cos \theta)}, \\
& \sin ((l-1) \theta+\psi(\theta))=\frac{g(\cos \theta)}{w(\cos \theta)},
\end{aligned}
$$

то формула (2.41) примет вид (2.38), где функция $w(x)$ определена формулой (2.42), а функция $\psi(\theta)$ равна

$$
\psi(\theta)=\operatorname{arctg} \frac{\cos \theta F_{l-1}(\cos \theta)-F_{l}(\cos \theta)}{\sin \theta F_{l-1}(\cos \theta)}-(l-1) \theta .
$$

При дополнительных требованиях на $F_{l-1}(x), F_{l}(x)$ функции $u_{n}(x)$ имеют на $[-1,1]$ полный альтернанс из $n+1$ точки [6], [7], [26].

Рассмотрим примеры построения оптимальных алгоритмов.

\section{§3. Интерполирование с весом}

Рассмотрим задачу о приближении интерполяционными многочленами Лагранжа $L_{n-1}(x)$ с $n$ узлами $x_{1}, x_{2}, \ldots, x_{n} \in[-1,1][1],[27]$ множества функций $F_{n} \subset$ $C^{n}[-1,1]$, характеризуемого условием $f(x) \in F_{n}$, если

$$
\left|f^{(n)}(x)\right| \leqslant R .
$$

Здесь $R>0$ - константа. 
Известно, что ошибка метода интерполяции $R_{n-1}(x)=f(x)-L_{n-1}(x)$ равна $f^{(n)}\left(\xi_{x}\right) \omega_{n}(x) / n$ ! , где

$$
\omega_{n}(x)=\prod_{i=1}^{n}\left(x-x_{i}\right), \quad \xi_{x} \in[-1,1]
$$

В пространстве $C[-1,1]$ для класса $F_{n}$ наилучшими узлами интерполяции будут $[5],[7]$

$$
x_{k}=\cos \frac{(2 k-1) \pi}{2 n}, \quad k=1, \ldots, n .
$$

Часто возникает аналогичная задача в пространстве $C([-1,1], w)$ с нормой $\|f\|=\max _{-1 \leqslant x \leqslant 1}|f(x) w(x)|$, решение которой дает наилучшее взвешенное на отрезке $[-1,1]$ приближение. Тогда ошибка метода на классе $F_{n}$ в метрике $C([-1,1], w)$ равна

$$
\sup _{f \in F_{n}} \sup _{-1 \leqslant x \leqslant 1}\left|\frac{f^{(n)}\left(\xi_{x}\right)}{n !} \omega_{n}(x) w(x, \alpha, \beta, C)\right|=\frac{R}{n !} \max _{-1 \leqslant x \leqslant 1}\left|\omega_{n}(x) w(x, \alpha, \beta, C)\right| .
$$

Поскольку inf $x_{1}, x_{2}, \ldots, x_{n} \max _{-1 \leqslant x \leqslant 1}\left|\omega_{n}(x) w(x, \alpha, \beta, C)\right|$ в $(3.3)$ равен $\widetilde{A}_{n}^{-1}$ и он достигается для корней $x_{i}$ многочлена $A_{n}^{-1} C_{n}(x, \alpha, \beta, C)$ - решении задачи I $(0.1)$, $(1.1),(1.4)$ среди многочленов вида $(3.2)$, то оптимальным многочленом $L_{n-1}(x)$ будет

$$
\begin{gathered}
L_{n-1}(x)=\sum_{i=1}^{n} \frac{(-1)^{i-1}\left(1-x_{i}^{2}\right)^{1 / 2} w\left(x_{i}, \alpha, \beta, C\right)}{N+\psi^{\prime}\left(\theta_{i}\right)} f\left(x_{i}\right) \frac{C_{n}(x, \alpha, \beta, C)}{x-x_{i}} \\
=\sum_{i=1}^{n} \frac{(-1)^{i-1} \sin \theta_{i} w\left(x_{i}, \alpha, \beta, C\right)}{N+\psi^{\prime}\left(\theta_{i}\right)} f\left(x_{i}\right) \frac{\cos (N \theta-\beta \pi+\psi(\theta))}{w(x, \alpha, \beta, C)\left(\cos \theta-\cos \theta_{i}\right)},
\end{gathered}
$$

где $\theta_{i}$ определены в $(2.7)$. Рассмотрим функцию $\lambda_{n}(x)$ и константу Лебега $\lambda_{n}$

$$
\begin{aligned}
\lambda_{n}(x) & =\sum_{i=1}^{n}\left|\frac{\left(1-x_{i}^{2}\right)^{1 / 2} w\left(x_{i}, \alpha, \beta, C\right)}{N+\psi^{\prime}\left(\theta_{i}\right)} \frac{C_{n}(x)}{x-x_{i}}\right| \\
& =\sum_{i=1}^{n}\left|\frac{\sin \theta_{i} w\left(x_{i}, \alpha, \beta, C\right)}{N+\psi^{\prime}\left(\theta_{i}\right)} \frac{\cos (N \theta-\beta \pi+\psi(\theta))}{w(x, \alpha, \beta, C)\left(\cos \theta-\cos \theta_{i}\right)}\right|
\end{aligned}
$$

$\lambda_{n}=\max _{x \in[-1,1]} \lambda_{n}(x)$. Имеет место

Лемма 2. Для кажсдого $i=1, \ldots, n$ справедливо неравенство

$$
J_{i}=\left|\frac{\sin \theta_{i} w\left(x_{i}, \alpha, \beta, C\right) \cos (N \theta-\beta \pi+\psi(\theta))}{\left(N+\psi^{\prime}\left(\theta_{i}\right)\right)\left(\cos \theta-\cos \theta_{i}\right)}\right| \leqslant \pi D g,
$$

$2 \partial e$

$$
g=\frac{\max _{\theta \in[0, \pi]}\left|1+\psi^{\prime}(\theta) / N\right|}{\min _{\theta \in[0, \pi]}\left|1+\psi^{\prime}(\theta) / N\right|}, \quad D=\max _{x \in[-1,1]} w(x, \alpha, \beta, C)
$$


ДокАЗАТЕЛЬСТво. Пусть для определенности $\theta \neq \theta_{i}$. Тогда

$$
\begin{gathered}
|\cos (N \theta-\beta \pi+\psi(\theta))|=\left|\cos (N \theta-\beta \pi+\psi(\theta))-\cos \left(N \theta_{i}-\beta \pi+\psi\left(\theta_{i}\right)\right)\right| \\
\quad \leqslant 2\left|\sin \frac{N\left(\theta-\theta_{i}\right)+\psi(\theta)-\psi\left(\theta_{i}\right)}{2}\right| \leqslant\left|\left(N\left(\theta-\theta_{i}\right)+\psi(\theta)-\psi\left(\theta_{i}\right)\right)\right|,
\end{gathered}
$$

a

$$
\left|\cos \theta-\cos \theta_{i}\right|=2\left|\sin \frac{\theta-\theta_{i}}{2} \sin \frac{\theta+\theta_{i}}{2}\right| \geqslant \frac{2}{\pi}\left|\left(\theta-\theta_{i}\right) \sin \frac{\theta+\theta_{i}}{2}\right| .
$$

Поэтому

$$
J_{i} \leqslant \frac{\pi D g}{2} \frac{\sin \theta_{i}}{\sin \left(\left(\theta+\theta_{i}\right) / 2\right)}
$$

Ho

$$
\frac{\sin \theta_{i}}{\sin \left(\left(\theta+\theta_{i}\right) / 2\right)} \leqslant \frac{\sin \theta_{i}+\sin \theta}{\sin \left(\left(\theta+\theta_{i}\right) / 2\right)}=2 \cos \frac{\theta-\theta_{i}}{2} \leqslant 2
$$

Лемма 2 доказана.

\section{Справедлива}

ТеОРема 3. Константа Лебега $\lambda_{n}$ для интерполячионных многочленов Лагранжа, построенных по корням $\left\{x_{j}^{n}\right\}$ для $\alpha=\beta=0$, оченивается сверху зависящей от $C$ величиной порядка $O(\ln n)$.

ДокАЗАТЕЛЬСТво теоремы 3 , используюшее лемму 2 , в основном совпадает с соответствуюшим доказательством для многочленов Чебышёва $T_{n}(x)[27]$. Если $\theta=\theta_{i}$, то $\lambda_{n}(x)=1$. Пусть теперь $\theta_{m}<\theta<\theta_{m+1}$. Тогда $\lambda_{n}(x)=\left(\sigma_{1}+\sigma_{2}\right) / w(x)$, где

$$
\begin{aligned}
\sigma_{1}= & \sum_{i=1}^{m-2}\left|\frac{\sin \theta_{i} w\left(x_{i}\right) \cos (N \theta-\beta \pi+\psi(\theta))}{\left(N+\psi^{\prime}\left(\theta_{i}\right)\right)\left(\cos \theta-\cos \theta_{i}\right)}\right| \\
& +\left|\frac{\sin \theta_{m-1} w\left(x_{m-1}\right) \cos (N \theta-\beta \pi+\psi(\theta))}{\left(N+\psi^{\prime}\left(\theta_{m-1}\right)\right)\left(\cos \theta-\cos \theta_{m-1}\right)}\right| \\
& +\left|\frac{\sin \theta_{m} w\left(x_{m}\right) \cos (N \theta-\beta \pi+\psi(\theta))}{\left(N+\psi^{\prime}\left(\theta_{m}\right)\right)\left(\cos \theta-\cos \theta_{m}\right)}\right|, \\
\sigma_{2}= & \sum_{i=m+1}^{n}\left|\frac{\sin \theta_{i} w\left(x_{i}\right) \cos (N \theta-\beta \pi+\psi(\theta))}{\left(N+\psi^{\prime}\left(\theta_{i}\right)\right)\left(\cos \theta-\cos \theta_{i}\right)}\right|,
\end{aligned}
$$

причем если $0<\theta<\theta_{1}$, то $\sigma_{1}=0$, а если $\theta_{n}<\theta<\pi$, то $\sigma_{2}=0$. Обе суммы оцениваются одинаково. Оценим первую сумму, предполагая, что $\theta_{1}<\theta$. При $m=1$ в $\sigma_{1}$ присутствует только третье слагаемое, а при $m=2$-второе и третье. В любом случае согласно лемме 2 их сумма не превышает $2 \pi D g$. При $m>2$ имеем

$$
\sigma_{1} \leqslant 2 \pi D g+D \sum_{i=1}^{m-2}\left|\frac{\sin \theta_{i}}{\left(N+\psi^{\prime}\left(\theta_{i}\right)\right)\left(\cos \theta-\cos \theta_{i}\right)}\right| .
$$

Функция $\sin x /(\cos x-\cos \theta)$ возрастает при $0<x<\theta$. Следовательно,

$$
\sin \frac{\theta_{i}}{\cos \theta_{i}-\cos \theta} \leqslant \sin \frac{x}{\cos x-\cos \theta}, \quad \theta_{i} \leqslant x \leqslant \theta_{i+1} .
$$


Отсюда

$$
\frac{\left(\theta_{i+1}-\theta_{i}\right) \sin \theta_{i}}{\cos \theta_{i}-\cos \theta} \leqslant \int_{\theta_{i}}^{\theta_{i+1}} \frac{\sin x d x}{\cos x-\cos \theta} .
$$

Из уравнений (2.7) следует, что

$$
\frac{\pi}{N}=\left(\theta_{i+1}-\theta_{i}\right)\left(1+\frac{\psi\left(\theta_{i+1}\right)-\psi\left(\theta_{i}\right)}{\left(\theta_{i+1}-\theta_{i}\right) N}\right),
$$

т.е. $\theta_{i+1}-\theta_{i}=\pi /\left(N+\psi^{\prime}\left(\theta_{i}^{\prime}\right)\right)$, где $\theta_{i} \leqslant \theta_{i}^{\prime} \leqslant \theta_{i+1}$. Пусть

$$
d=\frac{\max _{\theta \in[0, \pi]}\left(1+\left|\psi^{\prime}(\theta)\right| / N\right)}{\pi \min _{\theta \in[0, \pi]}\left(1+\left|\psi^{\prime}(\theta)\right| / N\right)}
$$

тогда

$$
\left|\frac{\sin \theta_{i}}{\left(N+\psi^{\prime}\left(\theta_{i}\right)\right)\left(\cos \theta-\cos \theta_{i}\right)}\right| \leqslant d \frac{\left(\theta_{i+1}-\theta_{i}\right) \sin \theta_{i}}{\cos \theta_{i}-\cos \theta} \leqslant d \int_{\theta_{i}}^{\theta_{i+1}} \frac{\sin x d x}{\cos x-\cos \theta} .
$$

Поэтому

$$
\sigma_{1} \leqslant 2 \pi D g+d \int_{0}^{\theta_{m-1}} \frac{\sin x d x}{\cos x-\cos \theta}=2 \pi D g+d \ln \frac{1-\cos \theta}{\cos \theta_{m-1}-\cos \theta} .
$$

Ho $1-\cos \theta \leqslant 2$,

$$
\cos \theta_{m-1}-\cos \theta>\cos \theta_{m-1}-\cos \theta_{m}=2 \sin \frac{\theta_{m-1}+\theta_{m}}{2} \sin \frac{\theta_{m-1}-\theta_{m}}{2},
$$

a

$$
\frac{\pi}{2 N} \leqslant \theta_{m-1}, \quad \theta_{m} \leqslant \pi-\frac{\pi}{2 N}, \quad \sin \frac{\theta_{m-1}+\theta_{m}}{2} \geqslant \sin \frac{\pi}{2 N} \geqslant \frac{1}{2 N},
$$

отсюда

$$
\sigma_{1} \leqslant 2 \pi D g-d \ln \sin \frac{\theta_{m-1}+\theta_{m}}{2}-d \ln \sin \frac{\theta_{m-1}-\theta_{m}}{2} \leqslant 2 \pi D g+2 d \ln N .
$$

Такие же оценки верны и для $\sigma_{2}$ ч.т.д.

\section{§4. Квадратурные формулы повышенной точности для некоторых классов интегралов с зависящими от параметров весовыми функциями}

Сушествуют связанные с именами Гаусса, Маркова, Лобатто, Радо квадратурные формулы повышенной алгебраической степени точности для приближенного вычисления интегралов с весом $p(x)[28],[29]$. Использование в них зависящих от параметров весовых функций $p(x, C)$ позволяет более точно учесть априорную информацию о свойствах класса интегрируемых фукнкций (примером служат задачи теории переноса частиц с узконаправленными индикатрисами рассеяния). Как правило, алгоритмы вычисления параметров таких квадратурных формул высоких порядков точности достаточно трудоемки [30], [31]. Однако существует такой 
класс весовых функций $p(x, C)(2.10)$, для которых сравнительно просто выгисляются параметры упомянутых квадратур. Это квадратурные формулы, узлы которых совпадают с корнями ортогональных ЧМБС-многочленов [32], [33]. Они обобшают знаменитую формулу Мелера [1], [28], [29]:

$$
\int_{-1}^{1} f(x) \frac{d x}{\sqrt{1-x^{2}}} \approx \frac{\pi}{n} \sum_{i=1}^{n} f\left(\cos \frac{(2 i-1) \pi}{2 n}\right) .
$$

Пусть $p(x)=p(x, \alpha, \beta, C)$ является функцией (2.10). Рассмотрим для выгисления интегралов вида

$$
I(f)=\int_{-1}^{1} f(x) p(x, \alpha, \beta, C) d x, \quad f(x) \in C[-1,1],
$$

алгоритмы определения параметров $A_{i}^{n}, x_{i}^{n}$ квадратурных формул

$$
I_{n}(f)=\sum_{i=1}^{n} A_{i}^{n} f\left(x_{i}^{n}\right)
$$

для весовых функций $p(x)(2.10)$. Точки $x_{i}^{n}$ называются узлами, а числа $A_{i}^{n}-$ весами квадратурной формуль (4.2). Эти параметры определяем из условия $I_{n}(f)=I(f)$, если $f(x)$ - многочлен степени не выше $M, n \leqslant M \leqslant 2 n-1$. Получим квадратурные формулы различного типа. В формулах для погрешности квадратур $R_{M}(f)$ участвуют производные $f^{(M+1)}(\eta)$, которые считаем непрерывными функциями на $[-1,1]$, а $\eta \in[-1,1]$. Нам понадобятся значения $C_{n}^{\prime}\left(x_{i}, \alpha, \beta, C\right) \times$ $C_{n+1}\left(x_{i}, \alpha, \beta, C\right)$ в корнях $x_{i}$ многочлена $C_{n}(x, \alpha, \beta, C)$. Используя $(1.5)$, получаeM

$$
C_{n}^{\prime}\left(x_{i}, \alpha, \beta, C\right) C_{n+1}\left(x_{i}, \alpha, \beta, C\right)=\frac{\left(N+\psi^{\prime}\left(\theta_{i}\right)\right) S_{l}\left(x_{i}\right)}{(1+x)^{2 \alpha}(1-x)^{2 \beta}}
$$

4.1. Приведем алгоритм определения параметров $A_{i}^{n}, x_{i}^{n}$ квадратурных формул типа Гаусса при весовых функциях вида $p(x)=w^{2}(x, \alpha, \beta, C)\left(1-x^{2}\right)^{-1 / 2}$. Пусть $N>\frac{l}{2}$, тогда согласно $(2.13),(2.19)$ многочлены $(1.17)$, умноженные на $\sqrt{2 / \pi}$, будут ортонормированными многочленами, у которых с ростом $n$ удваиваются коэффициенты $\widetilde{A}_{n}$ при степени $x^{n}(1.18)$. Следовательно [28], [29], $x_{i}^{n}=x_{i}-$ корни (2.7), a

$$
A_{i}^{n}=-\frac{\pi}{C_{n}^{\prime}\left(x_{i}, \alpha, \beta, C\right) C_{n+1}\left(x_{i}, \alpha, \beta, C\right)}, \quad i=\overline{1, n}
$$

или с учетом (4.3)

$$
A_{i}^{n}=\frac{\pi\left(1+x_{i}^{n}\right)^{2 \alpha}\left(1-x_{i}^{n}\right)^{2 \beta}}{S_{l}\left(x_{i}^{n}\right)\left(N+\psi^{\prime}\left(\theta_{i}\right)\right)}, \quad i=\overline{1, n} .
$$

Алгебраическая степень точности квадратуры (4.2), (4.5) $M=2 n-1$ и согласно [28], [29] с учетом (2.10), (3.2), (1.19) имеем

$$
R_{2 n-1}(f)=\frac{f^{(2 n)}(\eta)}{(2 n) !} \int_{-1}^{1} \omega_{n}^{2}(x) p(x, C) d x=\frac{f^{(2 n)}(\eta) \pi}{(2 n) ! 2^{2 N-1} B_{m}(C)}
$$




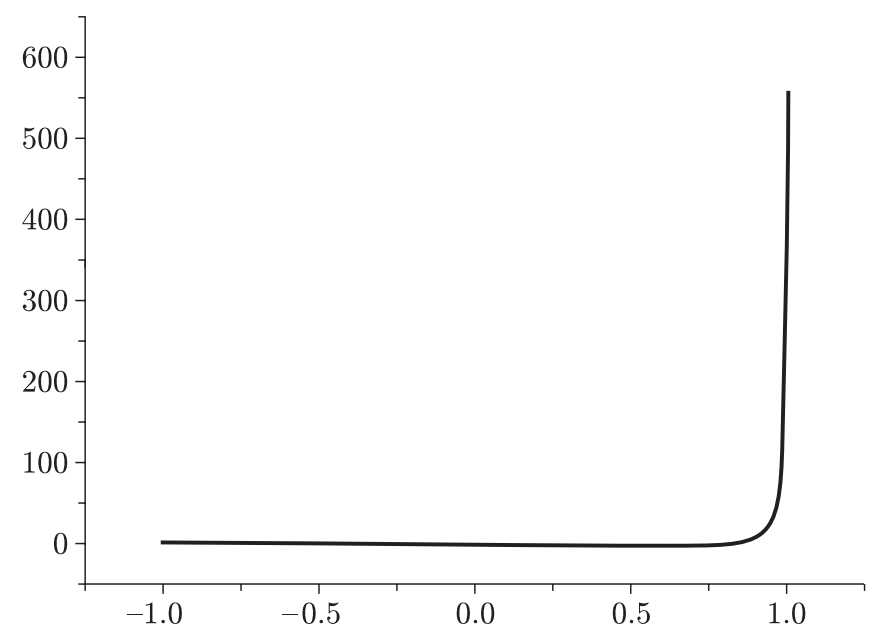

Рис. 9. Распределение коэффициентов $A_{i}^{40}$ квадратурной формулы Гаусса для (2.6)

ПримеР 4. Пусть $S_{l}(x)$ из (2.6), где $l=2, \delta=1.01$. На рис. 9 приведен график коэффициентов $A_{i}^{40}(4.5)$ квадратурной формулы Гаусса (4.2).

Теперь получим для рассматриваемого класса весовых функций явный вид параметров квадратурных формул типа Маркова, Лобатто, Радо, обобщая для этого случая результаты И.П. Мысовских [29] и используя из [28], [29] общие формулы для $A_{i}^{n}$. В них $\left\{x_{i}^{n}\right\}$ будут обозначать внутренние узлы отрезка $[-1,1]$, являющиеся корнями многочлена $C_{n}(x)=C_{n}\left(x, \alpha, \frac{1}{2}, C\right)$. Для вычисления весовых коэффициентов при $x= \pm 1$ использована формула Кристоффеля-Дарбу (2.14) и равенства $(2.15)-(2.18)$.

4.2. Рассмотрим формулы Лобатто-Радо. Пусть $\beta=0$, а квадратурная формула взята в виде

$$
I_{n}(f)=\sum_{i=1}^{n} A_{i}^{n} f\left(x_{i}^{n}\right)+A_{0}^{n} f(1) .
$$

Тогда в $(4.7)\left\{x_{i}^{n}\right\}$ - корни $C_{n}\left(x, \alpha, \frac{1}{2}, C\right)$ из ортогональной с весом $p\left(x, \alpha, \frac{1}{2}, C\right)$ системы многочленов.

Согласно [28], [29] для внутренних узлов справедливы формулы

$$
\begin{aligned}
A_{i}^{n} & =\frac{2}{D_{n+1}\left(x_{i}, 1, \alpha, 1 / 2\right) D_{n}^{\prime}\left(x_{i}, 1, \alpha, 1 / 2\right)\left(1-x_{i}\right)} \\
& =\frac{\pi}{C_{n}^{\prime}\left(x_{i}, \alpha, 1 / 2, C\right) C_{n+1}\left(x_{i}, \alpha, 1 / 2, C\right)\left(1-x_{i}\right)}, \quad i=\overline{1, n} .
\end{aligned}
$$

Тогда согласно (4.3)

$$
A_{i}^{n}=\frac{\pi\left(1+x_{i}^{n}\right)^{2 \alpha}}{S_{l}\left(x_{i}^{n}\right)\left(n+\alpha+1 / 2+\psi^{\prime}\left(\theta_{i}\right)\right)}, \quad i=\overline{1, n} .
$$

Для коэффициента $A_{0}^{n}$ в узле $x=1$ имеем

$$
A_{0}^{n}=\frac{1}{D(1,1, \alpha, 0)} \int_{-1}^{1} D_{n}(x, 1, \alpha, 0) p(x, \alpha, 0, C) d x,
$$


причем согласно $(2.16),(2,18),(4.5)$ он равен

$$
A_{0}^{n}=\frac{\pi 2^{2 \alpha-1}}{S_{l}(1)\left(n+\alpha+1 / 2+\psi^{\prime}(0)\right)},
$$

$M=2 n$. Согласно [28], [29], учитывая (2.16), (2.18), (4.5) и то, что

$$
(1-x) p(x, \alpha, 0, C)=p\left(x, \alpha, \frac{1}{2}, C\right)
$$

имеем

$$
\begin{aligned}
R_{2 n}(f) & =\frac{f^{(2 n+1)}(\eta)}{(2 n+1) !} \int_{-1}^{1} \omega_{n}^{2}(x) p\left(x, \alpha, \frac{1}{2}, C\right) d x \\
& =\frac{f^{(2 n+1)}(\eta) \pi}{(2 n+1) ! 2^{2(n+\alpha)} B_{m}(C)} .
\end{aligned}
$$

4.3. Пусть $\alpha=\beta=0$, а квадратурная формула Маркова взята в виде

$$
I_{n}(f)=\sum_{i=1}^{n} A_{i}^{n} f\left(x_{i}^{n}\right)+A_{0}^{n} f(1)+B_{0}^{n} f(-1) .
$$

Тогда в $(4.2)\left\{x_{i}^{n}\right\}$ - корни $C_{n}\left(x, \frac{1}{2}, \frac{1}{2}, C\right)$ из ортогональной с весом $p\left(x, \frac{1}{2}, \frac{1}{2}, C\right)$ системы многочленов. Согласно [28], [29] для внутренних узлов справедливы формулы

$$
A_{i}^{n}=-\frac{\pi}{C_{n}^{\prime}\left(x_{i}, 1 / 2,1 / 2, C\right) C_{n+1}\left(x_{i}, 1 / 2,1 / 2, C\right)\left(1-x_{i}^{2}\right)}, \quad i=\overline{1, n} .
$$

Вычисляя по (4.3) $C_{n+1}\left(x_{i}, \frac{1}{2}, \frac{1}{2}, C\right) C_{n}^{\prime}\left(x_{i}, \frac{1}{2}, \frac{1}{2}, C\right)$, получаем

$$
A_{i}^{n}=\frac{\pi}{S_{l}\left(x_{i}^{n}\right)\left(n+1+\psi^{\prime}\left(\theta_{i}\right)\right)}, \quad i=\overline{1, n} .
$$

Для коэффициента $A_{0}^{n}$ в узле $x=1$ [28] по (2.16), (2.18) имеем

$$
\begin{aligned}
A_{0}^{n} & =\frac{1}{2 D_{n}(1,1,1 / 2,0)} \int_{-1}^{1} D_{n}\left(x, 1, \frac{1}{2}, 0\right)(1+x) p(x, 0,0, C) d x \\
& =\frac{1}{2 D_{n}(1,1,1 / 2,0)} \int_{-1}^{1} D_{n}\left(x, 1, \frac{1}{2}, 0\right) p\left(x, \frac{1}{2}, 0, C\right) d x, \\
A_{0}^{n} & =\frac{\pi}{2 S_{l}(1)\left(n+1+\psi^{\prime}(0)\right)} .
\end{aligned}
$$

Для коэффициента $B_{0}^{n}$ в узле $x=-1$ аналогично получаем

$$
B_{0}^{n}=\frac{\pi}{2 S_{l}(-1)\left(n+1+\psi^{\prime}(\pi)\right)},
$$

$M=2 n+1$. Согласно [27], учитывая $(2.16),(2.18),(4.5)$ и то, что

$$
\left(1-x^{2}\right) p(x, 0,0, C)=p\left(x, \frac{1}{2}, \frac{1}{2}, C\right)
$$


имеем

$$
\begin{aligned}
R_{2 n+1}(f) & =\frac{f^{(2 n+2)}(\eta)}{(2 n+2) !} \int_{-1}^{1} \omega_{n}^{2}(x) p\left(x, \frac{1}{2}, \frac{1}{2}, C\right) d x \\
& =\frac{f^{(2 n+2)}(\eta) \pi}{(2 n+2) ! 2^{2 n+1} B_{m}(C)}
\end{aligned}
$$

4.4. Рассмотрим квадратурные формулы повышенной точности для вычисления в смысле главного значения сингулярных интегралов вида

$$
I(f, a)=\int_{-1}^{1} \frac{f(x) p(x, \alpha, \beta, C) d x}{x-a}=\int_{-1}^{1} \frac{(f(x)-f(a)) p(x, \alpha, \beta, C) d x}{x-a}+f(a) q(a)
$$

где $a \in(-1,1)$, функция $f(x) \in C[-1,1]$ удовлетворяет условию Гёльдера:

$$
|f(x)-f(y)| \leqslant L|x-y|^{\gamma}, \quad x, y \in[-1,1], \quad L>0, \quad 0<\gamma \leqslant 1,
$$

а функция Ляпунова-Стилтьеса $q(a)$ :

$$
q(a)=\int_{-1}^{1} \frac{p(x, \alpha, \beta, C) d x}{x-a}
$$

вычисляется явно в квадратурах для веса $p(x, \alpha, \beta, C)$ вида (2.10) [34].

Пусть $a$ не является корнем многочлена $C_{n}(x, \alpha, \beta, C)$, тогда квадратурную формулу возьмем в виде

$$
I_{n}(f, a)=\sum_{i=1}^{n} A_{i}^{n} \frac{f\left(x_{i}^{n}\right)-f(a)}{x_{i}^{n}-a}+f(a) q(a)
$$

В ней используем параметры (4.5) формулы Гаусса. Если же $а$ совпадает с одним из корней $\left(a=x_{j}^{n}\right)$, то, предполагая сушествование в этой точке производной от $f(x)$, заменяем в $j$-м слагаемом формулы (4.19) $\left(f\left(x_{j}^{n}\right)-f(a)\right) /\left(x_{j}^{n}-a\right)$ на $f^{\prime}\left(x_{j}^{n}\right)$. Здесь $M=2 n$.

Во втором методе воспользуемся следующим явно вычисляемым интегралом:

$$
\widetilde{q}(a, \alpha, \beta)=\int_{-1}^{1} \frac{(1+x)^{2 \alpha-1 / 2}(1-x)^{2 \beta-1 / 2} d x}{x-a},
$$

$\widetilde{q}(a, 0,0)=0, \widetilde{q}\left(a, \frac{1}{2}, 0\right)=-\widetilde{q}\left(a, 0, \frac{1}{2}\right)=\pi, \widetilde{q}\left(a, \frac{1}{2}, \frac{1}{2}\right)=\pi a$. Умножая (4.19) на $f(a) / S_{l}(a)$, преобразуем интеграл в равенстве $(4.18)$ :

$$
I(f, a)=\int_{-1}^{1} \frac{\left(f(x) S_{l}(a)-f(a) S_{l}(x)\right) p(x, \alpha, \beta, C) d x}{(x-a) S_{l}(a)}+\frac{f(a) \widetilde{q}(a, \alpha, \beta)}{S_{l}(a)} .
$$

Пусть $a$ не является корнем многочлена $C_{n}(x, \alpha, \beta, C)$, тогда квадратурная формула имеет вид

$$
I_{n}(f, a)=\frac{1}{S_{l}(a)}\left(\sum_{i=1}^{n} A_{i}^{n} \frac{f\left(x_{i}^{n}\right) S_{l}(a)-f(a) S_{l}\left(x_{i}^{n}\right)}{x_{i}^{n}-a}+f(a) \widetilde{q}(a, \alpha, \beta)\right) .
$$


В ней используем параметры (4.5) формулы Гаусса. Если $a$ совпадает с одним из корней $\left(a=x_{j}^{n}\right)$, то, предполагая сушествование в этой точке производной от $f(x)$, заменяем в $j$-м слагаемом формулы $(4.22)\left(f\left(x_{j}^{n}\right) S_{l}(a)-f(a) S_{l}\left(x_{j}^{n}\right)\right) /\left(x_{j}^{n}-a\right)$ на $S_{l}\left(x_{j}^{n}\right) f^{\prime}\left(x_{j}^{n}\right)-f\left(x_{j}^{n}\right) S_{l}^{\prime}\left(x_{j}^{n}\right)$. Алгебраическая степень точности квадратур (4.19), (4.22) $M=2 n$.

Квадратурные формулы пп. 4.1-4.4 обобщают формулу Мелера, и при $N>$ $\frac{l}{2}$ их можно трактовать как квадратурные формулы повышенной алгебраической точности, точно интегрирующие с весовой функцией $(1+x)^{2 \alpha-1 / 2}(1-x)^{2 \beta-1 / 2}$ дробно-рациональные от $x$ функции вида $f(x) / S_{l}(x)$, где $f(x)$ - многочлен степени не вьшше $M[32]$.

4.5. Квадратурные формулы наивысшей тригонометрической степени точности для интегрирования на отрезке $[0, \pi]$ четных периодических функций получаем из рассмотренных ранее после замены в интеграле (4.1) переменной интегрирования $x=\cos \theta$ и в квадратурных формулах $x_{i}^{n}=\cos \theta_{i}$. А именно, пусть

$$
\widetilde{f}(\theta)=f(\cos \theta), \quad \widetilde{S}_{l}(\theta)=S_{l}(\cos \theta)=\prod_{k=1}^{m}\left(1-c_{k} \cos \left(l_{k} \theta\right)\right),
$$

тогда интеграл (4.1) преобразуется в интеграл от уже не имеющей особенностей подьнтегральной функции:

$$
\widetilde{I}(\widetilde{f})=\int_{0}^{\pi} \widetilde{f}(\theta) \frac{(1+\cos \theta)^{2 \alpha}(1-\cos \theta)^{2 \beta}}{\widetilde{S}_{l}(\theta)} d \theta .
$$

Квадратурным формулам пा. 4.1-4.3 будут соответствовать квадратурные формулы для $\widetilde{I}(\widetilde{f})$, построенные по слегка неравномерной сетке узлов $\left\{\theta_{i}\right\}, 0, \pi$. Аналогичньм образом по (4.2), (4.5) строятся квадратурные формулы для сингулярных интегралов вида

$$
\widetilde{I}(\widetilde{f}, \varphi)=\int_{0}^{\pi} \widetilde{f}(\theta) \frac{(1+\cos \theta)^{2 \alpha}(1-\cos \theta)^{2 \beta}}{\widetilde{S}_{l}(\theta)(\cos \theta-\cos \varphi)} d \theta .
$$

Они, в частности, могут использоваться для вычисления асимптотической при $n \rightarrow \infty$ фазовой функции Бернштейна-Сегё [7] при $p(x)=t(x) / \sqrt{1-x^{2}}, t(x)>0$ :

$$
\bar{\psi}(\theta)=\frac{\sin \theta}{2 \pi} \int_{0}^{\pi} \frac{\ln (t(\cos \theta) / t(\cos \varphi))}{\cos \theta-\cos \varphi} d \varphi
$$

которая совпадает с $\psi(\theta)$ при $p(x)=p(x, 0,0, C)$.

4.6. Пусть $F(x)=f(x) p(x, \alpha, \beta, C)$, тогда

$$
I(f)=\int_{-1}^{1} F(x) d x
$$

и в квадратурной формуле Гаусса $(4.2),(4.5) A_{i}^{n} f\left(x_{i}^{n}\right)=C_{i}^{n} F\left(x_{i}^{n}\right)$, где

$$
C_{i}^{n}=\frac{\pi\left(1-\left(x_{i}^{n}\right)^{2}\right)^{1 / 2}}{N+\psi^{\prime}\left(\theta_{i}\right)}, \quad i=\overline{1, n} .
$$


В [32] автором было замечено, что весовые коэффициенты при $F\left(x_{i}^{n}\right)$ в квадратурных формулах трапеций, построенных по узлам квадратурных формул типа Гаусса $\left\{x_{i}^{n}\right\}$ и \pm 1 , близки к $C_{i}^{n}$. Покажем это. Коэффициент при $F\left(x_{i}^{n}\right), i=\overline{2, n-1}$, в формуле трапеций есть $B_{i}^{n}=\left(x_{i+1}^{n}-x_{i-1}^{n}\right) / 2$. Пользуясь формулами $(2.9)$, получаем, что

$$
B_{i}^{n}=\left(1-\cos ^{2}\left(\theta_{i}-\frac{\delta^{2} \psi\left(\theta_{i}\right)}{2 N}\right)\right)^{1 / 2} \sin \left(\frac{\pi}{N+\psi^{\prime}\left(\widetilde{\theta}_{i}\right)}\right)
$$

где $\delta^{2} \psi\left(\theta_{i}\right)=\psi\left(\theta_{i+1}\right)-2 \psi\left(\theta_{i}\right)+\psi\left(\theta_{i-1}\right), \theta_{i-1} \leqslant \widetilde{\theta}_{i} \leqslant \theta_{i+1}$. Таким образом, $B_{i}^{n}$ близки к $C_{i}^{n}$ при больших $n$. Изложенный подход использования $p(x, \alpha, \beta, C)$ и корней $C_{n}(x, \alpha, \beta, C)$ или асимптотически близких к ним величин (2.9) для построения узлов квадратурных формул трапеций может рассматриваться как дополнение к другим методам оптимизации параметров этих формул [35].

ПримеР 5. На рис. 10 приведено распределение коэффициентов $B_{i}^{40}, C_{i}^{40}$ для весовой функции (1.21), на рис. 11 - весовых коэффициентов $A_{i}^{40}$ квадратурной формулы Гаусса.

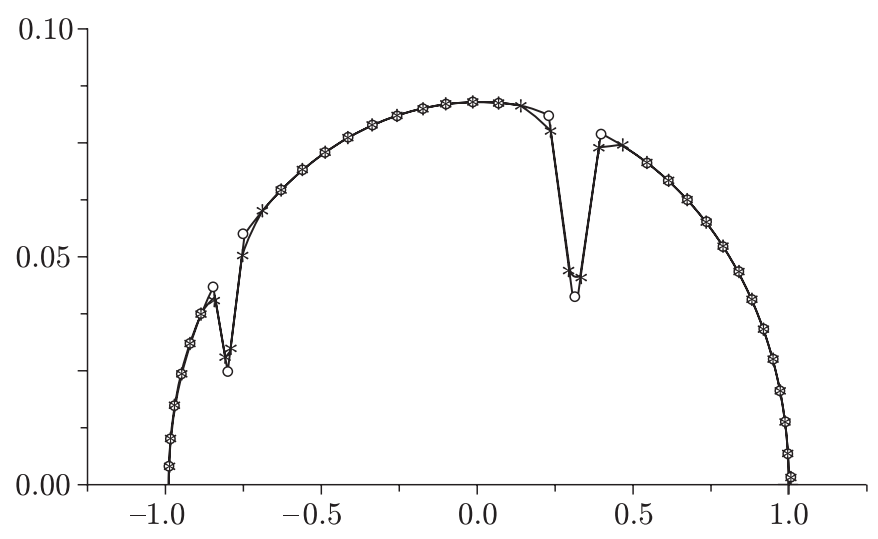

Рис. 10. Распределение коэффициентов $B_{i}^{40}$ формулы трапеций и коэффициентов $C_{i}^{40}$ из квадратурной формулы Гаусса: $*-B_{i}^{40}$, о $-C_{i}^{40}$ для $(1.21)$

4.7. Рассмотрим метод регуляризации интегралов с особенностью $(x-a)^{-2}$ в смысле конечной части по Адамару и квадратурных формул для них. Пусть в первых двух множителях $S_{l}(x)$ в (1.4) $l_{k}=1, c_{k}=\left(a+(-1)^{k} i \delta\right)^{-1}, a \in(-1,1)$, $k=1,2, \delta>0$,

$$
I(f, a, \delta)=\int_{-1}^{1}(f(x)-f(a)) p(x, \alpha, \beta, C) d x .
$$

Регуляризированным гиперсингулярным интегралом назовемпредел, если он существует: $I(f, a, 0)=\lim _{\delta \rightarrow 0} I(f, a, \delta)$. Каждому достаточно малому значению $\delta>0$ сопоставим квадратурную формулу Гаусса (4.2), (4.5):

$$
I_{n}(f, a, \delta)=\sum_{i=1}^{n} A_{i}^{n}\left(f\left(x_{i}^{n}\right)-f(a)\right)
$$




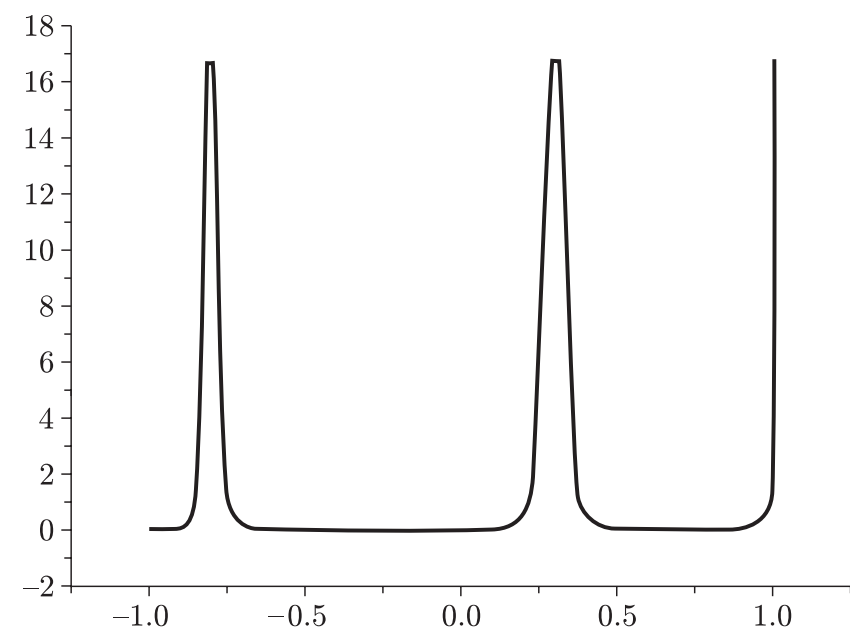

Рис. 11. Распределение коэффициентов $A_{i}^{40}$ квадратурной формулы Гаусса для (1.21)

которую используем для приближенного вычисления (4.28). После предельного перехода при $\delta \rightarrow 0$ в (4.29) возникают многочлены $C_{n}(x, \alpha, \beta, C)$, равные 0 при $x=a$ с разрывной в этой точке функцией $\psi(\theta)$, ортогональные с $p(x, \alpha, \beta, C)$ и экстремальные с $w(x, \alpha, \beta, C)$ - функцией с особенностью. Тогда для $I_{n}(f, a, 0)$ в (4.29) при достаточно гладкой функции $f(x)$ следует занулить слагаемое при $x_{j}=a$. Для простейшего случая

$$
p(x)=\frac{w^{2}(x)}{\left(1-x^{2}\right)^{1 / 2}}, \quad w(x)=\frac{\left(a^{2}+\delta^{2}\right)^{1 / 2}}{\left((x-a)^{2}+\delta^{2}\right)^{1 / 2}}
$$

рассмотрим

ПРимеР 6. Пусть $a=0.25, \delta=0.003$. На рис. 12-15 приведены графики: на рис. 12 - весовой функции $w(x)$, на рис. 13 - фазовой функции $\psi(\arccos x)$, на рис. 14 - ЧМБС-многочлена $C_{40}(x, 0,0, C)$, на рис. 15 - распределение коэффициентов $A_{i}^{40}(4.5)$ квадратурной формулы Гаусса (4.2).

Изложенный метод распространяется на случай для многомерных интегралов [11]. Другие методы регуляризации содержатся в [36].

\section{§5. Алгоритмы оптимизации чебышёвских итерационных методов на классах начальных ошибок}

Изучим оптимальные чебышёвские итерационные методы с зависящим от параметров весом. Это позволяет более точно учесть априорную информацию о начальной ошибке. Определим класс операторов с параметрами, характерный для операторов перехода, связьвающих ошибку $\varepsilon^{n}$ на $n$-м шаге с начальной ошибкой $\varepsilon^{0}$ в итерационных методах. Исследуем задачу минимизации нормы $\left\|\varepsilon^{n}\right\|_{1}$ относительно нормы $\left\|\varepsilon^{0}\right\|_{0}$. Рассмотрим случай, когда задача минимизации отношения этих норм решается явно с применением ЧМБС-многочленов. С помощью аппарата ЧМБС-многочленов покажем, что формулы для параметров зависят от того, к какому классу “гладкости” принадлежит $\varepsilon^{0}$. Этот факт был исследован в [14], 


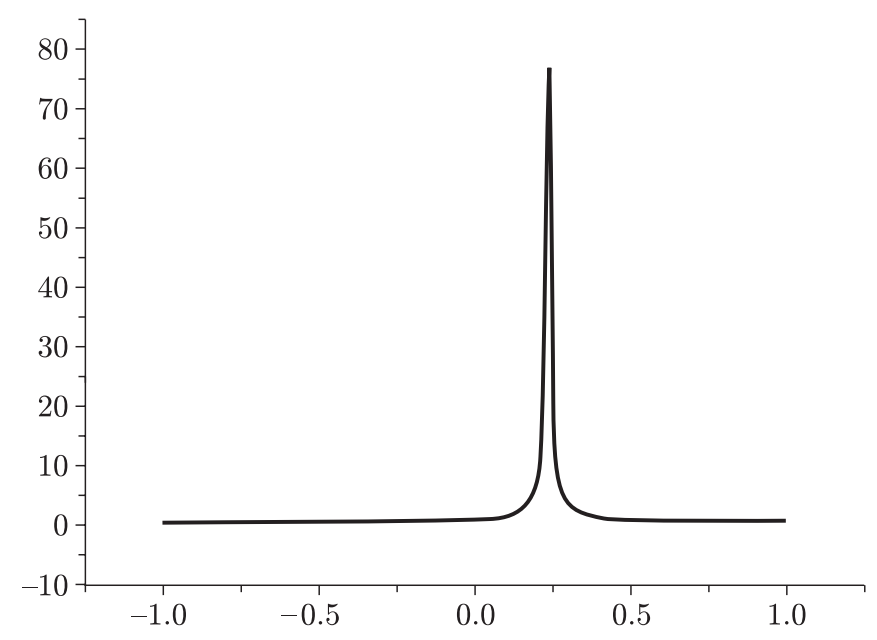

Рис. 12. Весовая функция $w(x)(4.30)$

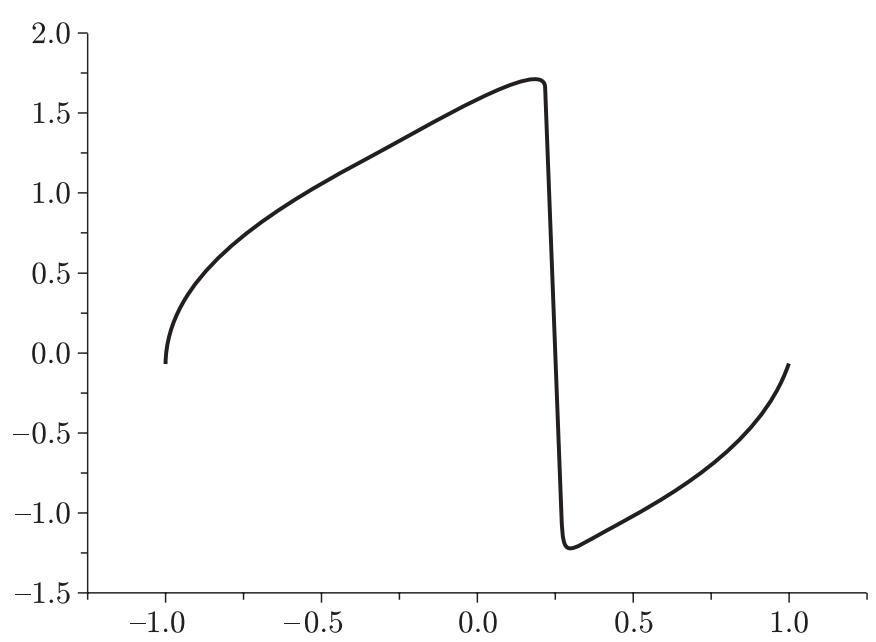

Рис. 13. Фазовая функция $\psi(\arccos x)$ для $(4.30)$

где было показано, что для "гладких" начальных ошибок возможно уменњшить их в конечное число раз независимо от обусловленности оператора решаемой задачи. Дадим формулы для параметров и точные оценки сходимости для оптимальных чебьшёвских методов в рассмотренных классах начальных ошибок.

Пусть $H$ - гильбертово пространство со скалярньм произведением $(\cdot, \cdot)$, $u, v \in H, A=A^{*}>0$ - самосопряженный положительно определенный оператор, $\left(\lambda_{i}, \varphi_{i}\right)$ - собственные пары оператора $A$. Причем $\operatorname{Sp}(A)$ - спектр оператора $A$ - принадлежит отрезку $[m, M], 0<m<M$, где $m, M$ - соответственно нижняя и верхняя границы спектра, $\lambda_{i} \leqslant \lambda_{i+1}, i=1,2, \ldots$, a $\left\{\varphi_{i}\right\}$ образуют ортонормированный базис в пространстве $H$. Определим функции от оператора $A$ следуюшим образом: если $f(\lambda)$ - ограниченная функция на $[m, M]$ и $u$ имеет разложение $u=\sum_{i} u_{i} \varphi_{i}$, то $f(A) u=\sum_{i} f\left(\lambda_{i}\right) u_{i} \varphi_{i}$. 


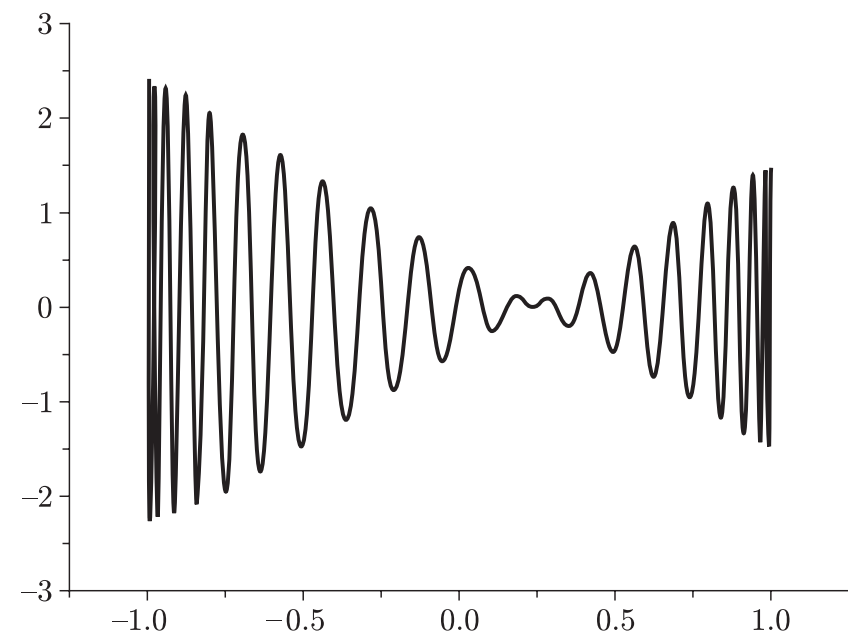

Рис. 14. ЧМБС-многочлен $C_{40}(x, 0,0, C)$ для (4.30)

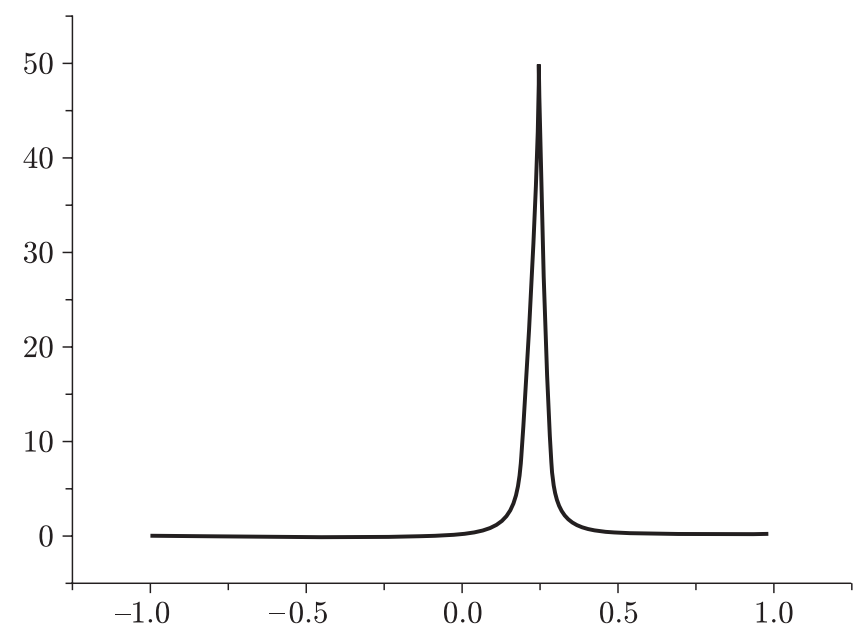

Рис. 15. Распределение коэффициентов $A_{i}^{40}$ квадратурной формулы Гаусса для $(4.30)$

Пусть $F(\lambda), G(\lambda), R(\lambda)$ - ограниченные на $[m, M]$ функции и $F(\lambda), G(\lambda)>0$ на $[m, M]$. Определим квадрат нормы:

$$
\|u\|_{F(A)}^{2}=(u, u)_{F(A)}=(F(A) u, u)=\sum_{i} F\left(\lambda_{i}\right) u_{i}^{2} .
$$

Пусть $v=q_{n}(A) R(A) u$, где $q_{n}(\lambda)$ - многочлен $n$-й степени вида

$$
q_{n}(\lambda)=\prod_{k=1}^{n}\left(1-\gamma_{k} \lambda\right), \quad q_{n}(0)=1
$$

Рассмотрим в $H$ эллипсоид $E$ :

$$
(u, u)_{G(A)}=\sum_{i} G\left(\lambda_{i}\right) u_{i}^{2}=D^{2}, \quad D>0 .
$$


Тогда

$$
(v, v)_{F(A)}=\left(q_{n}^{2}(A) R^{2}(A) F(A) u, u\right)=\left(q_{n}^{2}(A) W^{2}(A) u, u\right)_{G(A)},
$$

где $W(\lambda)=|R(\lambda)| \sqrt{F(\lambda) / G(\lambda)}$, и на множестве $E(5.1)$ имеем

$$
\|v\|_{F(A)} \leqslant \sup _{\lambda \in[m, M]}\left|q_{n}(\lambda) W(\lambda)\right| D .
$$

Пусть

$$
E_{n}=\inf _{q_{n}(0)=1} \sup _{\lambda \in[m, M]}\left|q_{n}(\lambda) W(\lambda)\right|, \quad P_{n}(\lambda)=\arg \inf _{q_{n}(0)=1} \sup _{\lambda \in[m, M]}\left|q_{n}(\lambda) W(\lambda)\right| .
$$

Тогда многочлен $P_{n}(\lambda)$ реализует

$$
\min _{q_{n}(0)=1} \sup _{A: \operatorname{Sp}(A) \in[m, M]}\|v\|_{F(A)} .
$$

Обозначим $s=m / M, \delta=(1+s) /(1-s), \tau=\left(1-s^{1 / 2}\right) /\left(1+s^{1 / 2}\right), t=2 /(M+m)$ и сделаем замену переменных

$$
\lambda=\lambda(x)=\left(1-\delta^{-1} x\right) t^{-1}, \text { или } x=x(\lambda)=\delta(1-t \lambda) .
$$

Тогда экстремальная задача (5.2), (5.3) перейдет в аналогичную задачу для $[-1,1](2.3),(2.4)$ с решением $(2.5)$ :

$$
p_{n}(x)=\frac{C_{n}(x)}{C_{n}(\delta)}, \quad e_{n}=\frac{1}{\left|C_{n}(\delta)\right|},
$$

где $w_{0} w(x)=W(\lambda(x))$, а $w_{0}$ - нормировочньй множитель, $e_{n}=E_{n} / w_{0}$.

Далее будем предполагать, что $\alpha=\beta=0, w(x)=1 / \sqrt{S_{l}(x)}$, т.е. $C_{n}(x)=$ $C_{n}(x, 0,0, C)$.

Оператор $G(A)$, определяюший эллипсоид (5.1), задан функцией

$$
G(\lambda)=F(\lambda)\left(\frac{R(\lambda)}{W(\lambda)}\right)^{2}=\frac{R^{2}(\lambda) F(\lambda) S_{l}(x(\lambda))}{w_{0}^{2}} .
$$

Рассмотрим простой, часто встречающийся в исследованиях случай, когда нормы формируются с помощью степени оператора $A: R(\lambda) \equiv 1, W(\lambda)=(m / \lambda)^{l / 2}$, $G_{l}(\lambda)=(\lambda / m)^{l} F(\lambda)$, где коэффициент $m$ взят для нормировки. Тогда

$$
\max _{[m, M]} W(\lambda)=1
$$

и упрошаются формулы для $C_{n}(\delta)$, так как

$$
S_{l}(x)=\left(1-\delta^{-1} x\right)^{l}, \quad \psi(\theta)=l \operatorname{arctg} \frac{\sin \theta}{\tau^{-1}-\cos \theta}, \quad E_{n}=(m t)^{l / 2} e_{n} .
$$

График $C_{n}(x, 0,0, C)$ будет “ужом" на отрезке $[-1,1]$ в коридоре, ограниченном ветвями парабол $\pm\left(1-\delta^{-1} x\right)^{l / 2}$ (см. пример 2 и рис. 4$)$. 
При этом корни $x_{i}$ будут возрастать с ростом $l$.

При $l=0$ имеем

$$
C_{n}(x)=T_{n}(x), \quad E_{n}=E_{0, n}=\frac{2 \tau^{n}}{1+\tau^{2 n}}
$$

А при $1 \leqslant l<2 n$

$$
\sqrt{1-\delta^{-1}} \operatorname{Co}_{1 / 2}(\delta)=\sqrt{1+\delta^{-1}} \operatorname{Si}_{1 / 2}(\delta)=\sqrt{\frac{2 m M}{M^{2}-m^{2}}}
$$

т.e.

$$
\begin{aligned}
& C_{n}(\delta)=\frac{\tau^{-n+l / 2}}{2}\left(\frac{8 m M}{M^{2}-m^{2}}\right)^{l / 2} \\
& E_{n}=E_{l, n}=2 \tau^{n-l / 2}\left(\frac{1-s}{4}\right)^{l / 2} .
\end{aligned}
$$

При $l=2$ этот случай был рассмотрен в [37], для него

$$
\begin{aligned}
C_{n}(x)= & \frac{1}{2 \delta}\left(\left(x+\sqrt{x^{2}-1}\right)^{n-1}\left(\delta x-1+\sqrt{\left(\delta^{2}-1\right)\left(x^{2}-1\right)}\right)\right. \\
& \left.+\left(x-\sqrt{x^{2}-1}\right)^{n-1}\left(\delta x-1-\sqrt{\left(\delta^{2}-1\right)\left(x^{2}-1\right)}\right)\right) .
\end{aligned}
$$

Заметим, что при $s=m / M<1, n, l \rightarrow \infty$

$$
\frac{E_{l, n}}{E_{0, n}}=\left(1+\tau^{2 n}\right)\left(\frac{1+s^{1 / 2}}{2}\right)^{l} \rightarrow 0
$$

Пусть в пространстве $H$ задано уравнение

$$
A u=f
$$

где $f \in H$. Если

$$
f=\sum_{i} f_{i} \varphi_{i}, \quad f_{i}=\left(f, \varphi_{i}\right)
$$

TO

$$
u=\sum_{i} \frac{f_{i} \varphi_{i}}{\lambda_{i}}
$$

Для нахождения решения (5.12) рассмотрим два типа итерационных методов: двучленньй

$$
u^{k+1}=u^{k}-\alpha_{k+1}\left(A u^{k}-f\right)
$$

и трехчленный

$$
u^{k+1}=u^{k}-\alpha_{k+1}\left(A u^{k}-f\right)-\beta_{k+1}\left(u^{k}-u^{k-1}\right),
$$


$k=0,1, \ldots, \beta_{1}=0$, в которых по заданному $u^{0}$ образуем последовательность $u^{k} \rightarrow u$. В (5.13), (5.14) $\alpha_{k}, \beta_{k}$ - набор параметров. Тогда [7]

$$
u^{n}=P_{n}(A) u^{0}+\left(I-P_{n}(A)\right) A^{-1} f
$$

где

$$
P_{n}(\lambda)=\prod_{i=1}^{n}\left(1-\gamma_{i} \lambda\right), \quad P_{n}(0)=1
$$

или

$$
A\left(I-P_{n}(A)\right)^{-1} u^{n}=f+A\left(I-P_{n}(A)\right)^{-1} P_{n}(A) u^{0},
$$

или

$$
A u^{n}=\left(I-P_{n}(A)\right) f+A P_{n}(A) u^{0} .
$$

Если

$$
\varepsilon^{k}=u-u^{k}=\sum_{i} \varepsilon_{i}^{k} \varphi_{i}
$$

то начальная ошибка $\varepsilon^{0}$ и $\varepsilon^{n}$ на $n$-м шаге связаны соотношением

$$
\varepsilon^{n}=P_{n}(A) \varepsilon^{0}=\sum_{i} P_{n}\left(\lambda_{i}\right) \varepsilon_{i}^{0} \varphi_{i}
$$

Коэффициенты многочлена $P_{n}(\lambda)$ вычисляются по параметрам каждого из методов (5.13), (5.14). Для метода (5.13)

$$
\gamma_{j_{k}}=\alpha_{k}=\frac{2}{M+m-(M-m) x_{j_{k}}}, \quad k=1,2, \ldots, n,
$$

где $x_{i}$ определены в $(2.7)$, а $\varkappa_{n}=\left(j_{1}, \ldots, j_{n}\right)$ - перестановка, задающая порядок употребления параметров [7]. Для метода (5.14) многочлен $P_{n}(\lambda)$ определяется последовательно:

$$
\begin{gathered}
P_{0}(\lambda)=1, \quad \beta_{1}=0, \quad P_{1}(\lambda)=1-\alpha_{1} \lambda, \\
P_{k+1}(\lambda)=\left(1-\beta_{k+1}-\alpha_{k+1} \lambda\right) P_{k}(\lambda)+\beta_{k+1} P_{k-1}(\lambda), \quad k=1, \ldots, n-1,
\end{gathered}
$$

где параметры определяются из формул для $\widetilde{C}_{1}(x)$ и рекуррентных соотношений типа (2.19), (2.20) после замены в них $x$ на $\lambda$ по формуле (5.4).

По формуле (5.13) реализуются двучленные чебышёвские методы различных типов [7], а по (5.14) - трехчленные чебышёвские методы, методы сопряженных градиентов, комбинированные итерационные методы [14], [17], [26].

Определим параметры оптимального метода при $k>\frac{l}{2}$. Для этого потребуем, чтобы многочлены $P_{k}(\lambda)$ удовлетворяли условию (5.16) и, будучи определенными соотношениями (5.21), были одновременно многочленами, наименее отклоняюшимися от нуля на отрезке $[m, M]$. Тогда согласно $(2.21)$ имеем

$$
P_{k}(\lambda)=\frac{C_{k}(x)}{C_{k}(\delta)}
$$


где $x$ получен по формулам (5.4). Определяя из $(5.22) C_{k}(x)$ и подставляя эти значения и величину $x$ из формулы (5.4) в соотношения $(5.20),(2.21)$, получаем

$$
\begin{gathered}
C_{1}(\delta) P_{1}(\lambda)=C_{1}\left(\frac{M+m-2 \lambda}{M-m}\right) \\
C_{k+1}(\delta) P_{k+1}(\lambda)=2\left(\frac{M+m-2 \lambda}{M-m}\right) C_{k}(\delta) P_{k}(\lambda)-C_{k-1}(\delta) P_{k-1}(\lambda) .
\end{gathered}
$$

Из сравнения формул (5.20), (5.21) с формулами (5.23) следует, что

$$
\alpha_{k+1}=\frac{4}{M-m} \frac{C_{k}(\delta)}{C_{k+1}(\delta)}, \quad \beta_{k+1}=-\frac{C_{k-1}(\delta)}{C_{k+1}(\delta)}, \quad k>\frac{l}{2} .
$$

При этих значениях параметров метод (5.14) становится оптимальным.

Разберем интересные частные случаи. Приведем характеристики оптимальных чебьшёвских методов. Для заданного $l$ и $S_{l}(x), \psi(\theta)$ из (5.7) определяем $E_{l, n}$ из $(5.8),(5.10), x_{i}$ из $(2.7), \gamma_{j_{k}}$ из $(5.19), a_{2}, b_{2}, d_{2}$ из $(2.20)$ по $C_{1}(x), C_{2}(x)$, $\alpha_{1}$ в $(5.14),(5.20)$ через корень $C_{1}(x)$, а остальные $\alpha_{k}, \beta_{k}$ в $(5.14),(5.21)$ определяем из (5.24) и уравнений типа (2.20).

1) Пусть $l=0, G(\lambda)=F(\lambda)$, тогда $w(x) \equiv 1, C_{n}(x)=T_{n}(x), a_{2}=2, b_{2}=0$, $d_{2}=1, C_{1}(x)=x, \alpha_{1}=t_{\text {и }}$

$$
\left\|\varepsilon^{n}\right\|_{F(A)} \leqslant E_{0, n}\left\|\varepsilon^{0}\right\|_{F(A)}=\frac{2 \tau^{n}}{1+\tau^{2 n}}\left\|\varepsilon^{0}\right\|_{F(A)} .
$$

$2)$ При $1 \leqslant l \leqslant 2 n$ пусть $l_{k}=1, G(\lambda)=G_{l}(\lambda)=F(\lambda)(\lambda / m)^{l}$ и из принадлежности $\varepsilon^{0}$ классу (5.1) согласно (5.10) получаем

$$
\left\|\varepsilon^{n}\right\|_{F(A)} \leqslant 2 \tau^{n-l / 2}\left(\frac{1-s}{4}\right)^{l / 2}\left\|\varepsilon^{0}\right\|_{G_{l}(A)} .
$$

Пусть коэффициенты $\varepsilon_{i}^{0}$ в разложении начальной ошибки $\varepsilon^{0}$ достаточно быстро убывают с ростом $i$, точнее: существует такая минимальная постоянная $0<$ $c(l)<1$, что $2^{1-l}\left\|\varepsilon^{0}\right\|_{G_{l}(A)} \leqslant c(l)\left\|\varepsilon^{0}\right\|_{F(A)}$ при $n \geqslant \frac{l}{2}$. Тогда $\left\|\varepsilon^{n}\right\|_{F(A)}$ уменьшится по крайней мере в $1 / c(l)$ раз по сравнению с аналогичной нормой начальной ошибки независимо от обусловленности оператора $A$. Рассмотрим частные случаи.

3) Пусть $l=1, G_{1}(\lambda)=F(\lambda) \lambda / m$ (такая норма для начальной ошибки характерна для методов сопряженных градиентов [14]):

$$
\left\|\varepsilon^{n}\right\|_{F(A)} \leqslant E_{1, n}\left\|\varepsilon^{0}\right\|_{G_{1}(A)}=\tau^{n-1 / 2}(1-s)^{1 / 2}\left\|\varepsilon^{0}\right\|_{G_{1}(A)},
$$

если $c=\left(1+s^{1 / 2}\right)(2(1+s))^{-1 / 2}$, то $a_{2}=2, b_{2}=\tau, d_{2}=c\left(1-\tau^{2}\right), C_{1}(x)=c(x-\tau)$, $C_{2}(x)=c\left(2 x^{2}-\tau x-1\right)$ и $\alpha_{1}=1 / \sqrt{M m}$.

4) Пусть $l=2, G_{2}(\lambda)=F(\lambda)(\lambda / m)^{2}$. В этом случае в чебышёвских методах минимизируется норма ошибки на $n$-м шаге по сравнению с нормой вычисляемой начальной невязки $r^{0}=f-A u^{0}=A \varepsilon^{0}$ :

$$
\left\|\varepsilon^{n}\right\|_{F(A)} \leqslant E_{2, n}\left\|\varepsilon^{0}\right\|_{G_{2}(A)}=\tau^{n-1} \frac{1-s}{2 m}\left\|r^{0}\right\|_{F(A)} .
$$


Если $\widetilde{c}=2 s^{1 / 2} /(1+s)$, то $a_{2}=1+\widetilde{c}, b_{2}=\widetilde{c} \delta^{-1}, d_{2}=2 s \widetilde{c}, C_{1}(x)=x-\delta^{-1}$, $C_{2}(x)=\left(\tau^{-1} x^{2}-x-\sqrt{\delta^{2}-1}\right) \delta^{-1}, \alpha_{1}=\left(m^{-1}+M^{-1}\right) / 2$. Этот метод был предложен в [38].

5) Рассмотрим следуюшую задачу II оптимизации (0.3). Пусть в итерационных методах (5.13), (5.14) взято $u^{0}=0$, тогда согласно (5.17) $u^{n}$ является решением уравнений

$$
A\left(I-P_{n}(A)\right)^{-1} u^{n}=f, \text { или } A u^{n}=\left(I-P_{n}(A)\right) f,
$$

a

$$
\varepsilon^{n}=P_{n}(A) u=P_{n}(A) A^{-1} f .
$$

Пусть $G(\lambda)=G_{l}(\lambda)$, тогдаметод, оптимальный еше и по параметру $l$, определим условием

$$
\left\|\varepsilon^{n}\right\|_{F(A)} \leqslant \min _{l \geqslant 0}\left(E_{l, n}\|u\|_{G_{l}(A)}\right)=\min _{l \geqslant 0}\left(\frac{E_{l, n}}{m}\|f\|_{G_{l-2}(A)}\right) .
$$

6) Пусть оператор $A$ имеет еше одну (искомую) собственную пару $\left(\lambda_{0}, \varphi_{0}\right)$, где $0<\lambda_{0}<m$. Рассмотренные в 1$)-5$ ) итерационные методы могут быть известньм способом преобразованы [3], [17] в итерационные методы для нахождения минимального изолированного собственного значения $\lambda_{0}$ и соответствуюшего собственного элемента $\varphi_{0}$. При этом для $l>0$ будет несколько усилен по сравнению с методом при $l=0$ эффект отделимости искомого собственного значения от соседних на классе начальных ошибок (5.1) при $G(\lambda)=G_{l}(\lambda)$. Например, для заданных $l, n$ и начального приближения

$$
u^{0}=u_{0} \varphi_{0}+\sum_{i} u_{i} \varphi_{i}, \quad u_{0} \neq 0
$$

двучленньй чебьшёвский метод с точностью до нормировки приближений для $\varphi_{0}$ имеет вид (5.13) при $f=0$ :

$$
u^{k+1}=u^{k}-\alpha_{k+1} A u^{k}, \quad k=0,1, \ldots, n-1 .
$$

Рассмотрим оптимальный смешанньй метод, состоящий из чебышёвских и обратных итераций. Пусть в (5.7) $l=2 q+p$, где величина $p$ характеризует класс начального приближения, а $Q(x)=C_{n}(x, 0,0, C) /\left(1-\delta^{-1} x\right)^{q}$. Тогда согласно (1.5) дробно-рациональная функция $Q(x)$ удовлетворяет уравнению

$$
\frac{Q(x)}{\left(1-\delta^{-1} x\right)^{p / 2}}=\cos (n \theta+\psi(\theta))
$$

Новое приближение с точностью до множителя к $\varphi_{0}$ получим по формуле

$$
u^{n+q}=Q(x(A)) u^{0} .
$$

Для этого продолжим цикл $q$ итерациями метода обратных итераций

$$
A u^{k+1}=u^{k}, \quad k=n, \ldots, n+q-1 .
$$


Общий порядок в объединенном цикле (5.28), (5.29) может быть произвольным. После $n+q$ итераций (5.28), (5.29) имеем формулу

$$
u^{n+q}=Q\left(x\left(\lambda_{0}\right)\right) u_{0} \varphi_{0}+\sum_{i} Q\left(x\left(\lambda_{i}\right)\right) u_{i} \varphi_{i}
$$

в которой существенно по сравнению с другими увеличился по модулю коэффициент при $\varphi_{0}$.

7) Комбинированные итерационные методы. Сначала решаем задачу (5.12) методом сопряженных градиентов (5.14) [17], сделав $l$ итераций и получив при этом информацию о распределении начальной ошибки в виде многочленов $F_{l-1}(x)=$ $P_{l-1}(x), F_{l}(x)=P_{l}(x)$, а затем, используя эту информацию для построения функции $w(x)$ вида $(2.42)$, переходим на трехчленный чебышёвский с весом $w(x)$ метод (5.14) [17], [26].

\section{§6. Алгоритмы оптимизации итерационных параметров в методе Федоренко-Бахвалова}

Изложенные алгоритмы могут быть эффективно использованы также при реализации циклов итераций на разных уровнях в методе Федоренко-Бахвалова (многосеточном методе) [1], [39], [40] или в аналогичном на операторном уровне КР-методе [17] с учетом на каждом уровне характера возникающих начальных ошибок и невязок. Для ускорения итераций в [40] были использованы параметры чебьшёвских многочленов, а в [17] - многочленов Якоби специального вида $-P_{n}^{(\alpha, n)}(x)$. Для прояснения сути этих методов рассмотрим идеальный для этого случая модельный пример методов подобного типа с коммутативньми операторами, когда собственные пары вспомогательных задач являются таковыми и для исходной задачи. При этом удобно использовать простейшие ЧМБС-многочлены явного вида $(2.25)$.

Пусть $0<g<1, m_{j}=g^{j} M, j=0,1, \ldots, J, m_{J}>m=m_{J+1}, H_{0}=H$, $A_{0}=A$, а $H_{j}$ - конечномерное подпространство, состоящее из элементов вида $\sum_{\lambda_{i} \leqslant m_{j}} a_{i} \varphi_{i}, Q_{j}$-ортопроектор на $H_{j}, Q_{j}^{1}=I-Q_{j}, j=1, \ldots, J, Q_{0}=Q_{J+1}^{1}=$ $F(A)=I, Q_{j}^{2}=Q_{j+1}^{1} Q_{j}, \widetilde{\delta}_{j}=\left(1+m_{j+1} / m_{j}\right) /\left(1-m_{j+1} / m_{j}\right), \lambda_{j}(x), x_{j}(\lambda)-$ функции $\lambda(x), x(\lambda)(5.4)$ при $\delta=\widetilde{\delta}_{j}, j=0,1, \ldots, J$, отображаюшие друг на друга отрезки $\left[m_{j+1}, m_{j}\right],[-1,1], R_{-1}=1$. Излагаемьй итерационный метод будет двухуровневьм. Внешние итерации состоят из $J+1$ блока. В каждом $j$-м блоке, $j=0,1, \ldots, J$, применяется итерационный метод (5.13) с $A=A_{j}$ длины $n_{j}$, $n_{j} \geqslant n_{j+1}$, с параметрами

$$
\alpha_{j_{k}}^{j}=\alpha_{k}^{j}=\frac{2}{m_{j}+m_{j+1}-\left(m_{j}-m_{j+1}\right) x_{j_{k}}^{j}}, \quad k=1,2, \ldots, n_{j}
$$

где $x_{j_{k}}^{j}$ - корни многочлена $\bar{C}_{n_{j}}\left(x, c_{j}, \bar{d}_{j}\right)(2.25)$, определяемого следующим образом. Пусть $P_{n_{j}}(\lambda)=\bar{C}_{n_{j}}\left(x_{j}(\lambda), c_{j}, \bar{d}_{j}\right) / \bar{C}_{n_{j}}\left(\delta_{j}, c_{j}, \bar{d}_{j}\right), \lambda \in\left[0, m_{j}\right],-$ многочлен оператора перехода в $j$-м блоке итераций $\left(P_{n_{j}}(0)=1\right)$, тогда

$$
0<P_{n_{j}}(\lambda) \leqslant \frac{T_{n_{j}}\left(x_{j}(\lambda)\right)}{T_{n_{j}}\left(\delta_{j}\right)} \leqslant\left(1-\frac{\lambda}{m_{j}}\right)^{n_{j}} \leqslant 1, \quad \lambda \in\left[0, m_{j+1}\right] .
$$


Пусть $R_{j}(\lambda)=P_{n_{j}}(\lambda) R_{j-1}(\lambda)$. Параметры $c_{j}, \bar{d}_{j}$ определяются по формулам (2.30), (2.31) по функции $v(x)(2.28)$, в которой $R(x)=R_{j-1}\left(\lambda_{j}(x)\right)$. Длину цикла $n_{j}$ определяем из условия

$$
\eta_{j}=e_{n}\left(c_{j}, \bar{d}_{j}\right) R_{j-1}\left(m_{j+1}\right) \leqslant \eta,
$$

где $e_{n}(c, d)$ определено в (2.27), а $\eta$ - точность решения. Расчет величин $n_{j}$ может быть сделан до начала итераций. Двусторонние оценки (2.33) для $\widetilde{e}_{n}$ позволяют для заданного $g$ оценить снизу величины $n_{j}$ на классе решаемых задач $(5.12)$ со спектром на $[m, M]$. В самом деле, если в проведенных рассуждениях положить $P_{n_{j}}(\lambda)=\bar{C}_{n_{j}}\left(x_{j}(\lambda), c_{j}, \widetilde{d}_{j}\right) / \bar{C}_{n_{j}}\left(\delta_{j}, c_{j}, \widetilde{d}_{j}\right), \lambda \in\left[0, m_{j}\right]$, то корни $x_{j_{k}}^{j}$ возрастут при $j \geqslant 1, R_{j}(\lambda)$ уменьшатся и оценка, подобная (6.3), будет выполнена при $\widetilde{n}_{j} \leqslant n_{j}$. Однако это не гарантирует решения с заданной точностью задач $(2.29)$ при $j=$ $1, \ldots, J$.

Обозначим через $u_{j}$ итерационное решение, полученное после реализации $j$-го итерационного блока.

Используя многочлен $\bar{C}_{n_{0}}(x, 0,0)=T_{n_{0}}(x)$ для нахождения приближенного решения уравнения (5.12) итерационным методом (5.13) длины $n_{0}$, получаем по формулам (5.13), (6.1) $u_{0}$ и ошибку $\varepsilon_{0}=u-u_{0}$

$$
\varepsilon_{0}=P_{n_{0}}(A) \varepsilon^{0}=\sum_{i} P_{n_{0}}\left(\lambda_{i}\right) \varepsilon_{i}^{0} \varphi_{i}
$$

которая удовлетворяет уравнению

$$
A \varepsilon_{0}=f-A u_{0}
$$

Опишем алгоритм дальнейших вычислений при $j=1$, который затем повторим при $j=2, \ldots, J$. Заменим уравнение (6.5) более простым приближенным заданным в $H_{1}$ конечномерным уравнением

$$
A_{1} w_{1}=Q_{1}\left(f-A u_{0}\right), \text { или } A_{1} w_{1}=Q_{1} A \varepsilon_{0},
$$

тогда $\varepsilon_{0}-w_{1}=Q_{1}^{1}\left(\varepsilon_{0}\right)$.

Используя для нахождения приближенного решения уравнения (6.6) итерационный метод (5.13) с $A=A_{1}, f=Q_{1}\left(f-A u_{0}\right)$ длины $n_{1} \leqslant n_{0}$, начиная с нулевого начального приближения, получаем $w_{1}$ по формулам (5.13), (5.25), (6.1)

$$
w_{1}=\left(I-P_{n_{1}}\left(A_{1}\right)\right) Q_{1} \varepsilon_{0},
$$

новое приближение $u_{1}=u_{0}+w_{1}$ и ошибку $\varepsilon_{1}$

$$
\begin{aligned}
\varepsilon_{1} & =u-u_{0}-w_{1}=Q_{1} P_{n_{1}}(A) P_{n_{0}}(A) \varepsilon^{0}+Q_{0}^{2} P_{n_{0}}(A) \varepsilon^{0} \\
& =Q_{0}^{2} P_{n_{0}}(A) \varepsilon^{0}+Q_{1}^{2} P_{n_{1}}(A) P_{n_{0}}(A) \varepsilon^{0}+Q_{2} P_{n_{1}}(A) P_{n_{0}}(A) \varepsilon^{0},
\end{aligned}
$$

которая удовлетворяет уравнению

$$
A_{1} \varepsilon_{1}=Q_{1}\left(f-A u_{1}\right) .
$$


Используем многочлен $\bar{C}_{n_{1}}\left(x, c_{1}, \bar{d}_{1}\right)$ для нахождения приближенного решения уравнения (6.7) итерационньм методом (5.13) длины $n_{1}$.

Продолжим для $j=2, \ldots, J$ изложенный итерационный метод. Используя для нахождения приближенного решения уравнения (6.6) итерационный метод (5.13) с $A=A_{j}, f=Q_{j}\left(f-A u_{j-1}\right)$ длины $n_{j} \leqslant n_{j-1}$, начиная с нулевого начального приближения, по формулам (5.13), (5.25), (6.1) получаем, что поправка $w_{j}$ удовлетворяет уравнению

$$
A_{j} w_{j}=Q_{j}\left(f-A u_{j-1}\right), \text { или } A_{j} w_{j}=Q_{j} A \varepsilon_{j-1}
$$

и $u_{j}=u_{j-1}+w_{j}$. Тем самым получаем генерирующий экстремальный с весом на отрезке $\left[m_{j+1}, m_{j}\right]$ ЧМБС-многочлен $P_{n_{j}}(\lambda)$ с параметрами $\alpha_{j_{k}}^{j}=\alpha_{k}^{j}, k=$ $1,2, \ldots, n_{j}(6.1)$.

При $j=J$ общая ошибка будет иметь вид

$$
\varepsilon_{J}=\left(\sum_{j=0}^{J} B_{j}\right) \varepsilon^{0}, \quad\left\|\varepsilon_{J}\right\| \leqslant \max _{j}\left\|B_{j}\right\|\left\|\varepsilon^{0}\right\|,
$$

где

$$
B_{j}=Q_{J}^{2} \prod_{k=0}^{j} P_{n_{k}}(A), \quad\left\|B_{j}\right\| \leqslant \eta .
$$

Для нашего модельного (идеального) алгоритма на этом итерации заканчиваются. В обшем случае, когда операторы $A_{j}$ не коммутируют (тогда собственные пары вспомогательных задач не совпадают с собственньпи парами исходной) и в реализации метода присутствуют алгоритмы интерполяции и восполнения, описанную итерационную процедуру повторяют. Полученные оценки для длин циклов можно рассматривать как оценки снизу для аналогичных алгоритмов в общем случае.

Рассмотрим характеристики предложенного метода применительно к сеточным эллиптическим задачам, в которых операторы $A_{n}$ построены на сетках с последовательным удвоением шага. Тогда $g=\frac{1}{4}$, т.е. $\delta=\frac{5}{3}, \tau_{10}=3$. Возьмем $\eta=10^{-4}$. Расчеты по формулам (2.23)-(2.32), (6.1), (6.3) показали, что $n_{0}=10, n_{j}=6$, $j=1,2 \ldots$, при этом с ростом $j$ величины $c_{j}, \widetilde{d}_{j}(0)$ быстро стремятся к предельным значениям: $c=0.66, d=0.52$, a $x_{i}^{j} \rightarrow x_{i}$ : 0.98178980, 0.83768660, 0.55206593, $0.11888926,-0.44026913,-0.92228107$. Затем был повторен расчет с этими предельными параметрами при $j>0$ и параметрами, равными корням $T_{10}(x)$ при $j=0$. Он дал то же распределение числа итераций с такими значениями: $3.410^{-5} \leqslant$ $\eta_{i} \leqslant 6.910^{-5}, \tau_{10}=3, \tau_{1 j}=4.5364, j=1,2, \ldots$ На рис. 16 показаны графики $w(x, c, d, 6)(2.23)$ и $v(x)(2.28)$ при $j=6$, на рис. 17 - график многочлена $\bar{C}_{6}(x, c, d)(2.25)$. Эти параметры можно рекомендовать для применения в методе Федоренко-Бахвалова.

\section{§7. Плохо обусловленные задачи с компактными операторами}

В разнообразных случаях предполагалось, что оператор $A$ в уравнении (5.12) таков, что в решении его, разложенном в ряд Фурье, основную роль играют члены, соответствующие минимальным собственным значениям. Однако существуют и другие случаи [41]. 


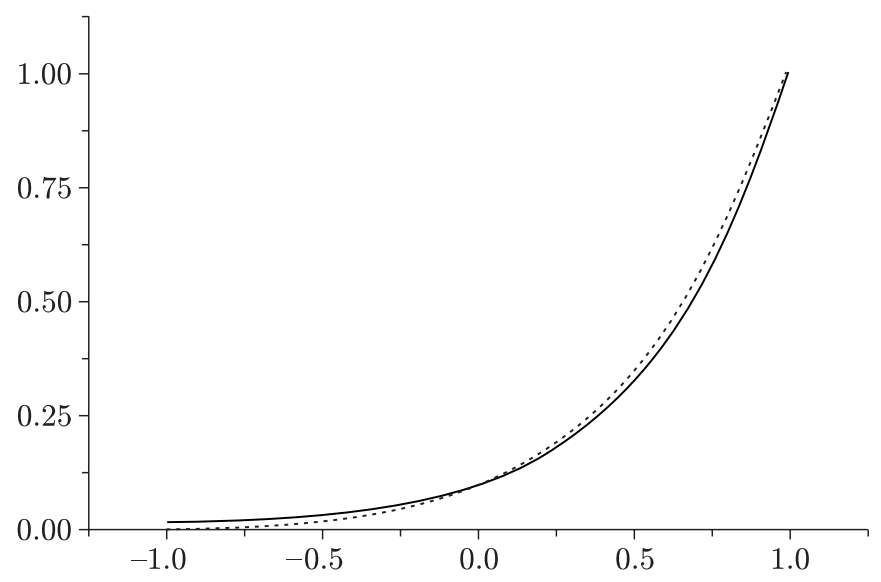

Рис. 16. Весовые функции: $w(x, c, d, 4)(2.23) \cdots \cdots \cdots \cdot ;(x)(2.28)$

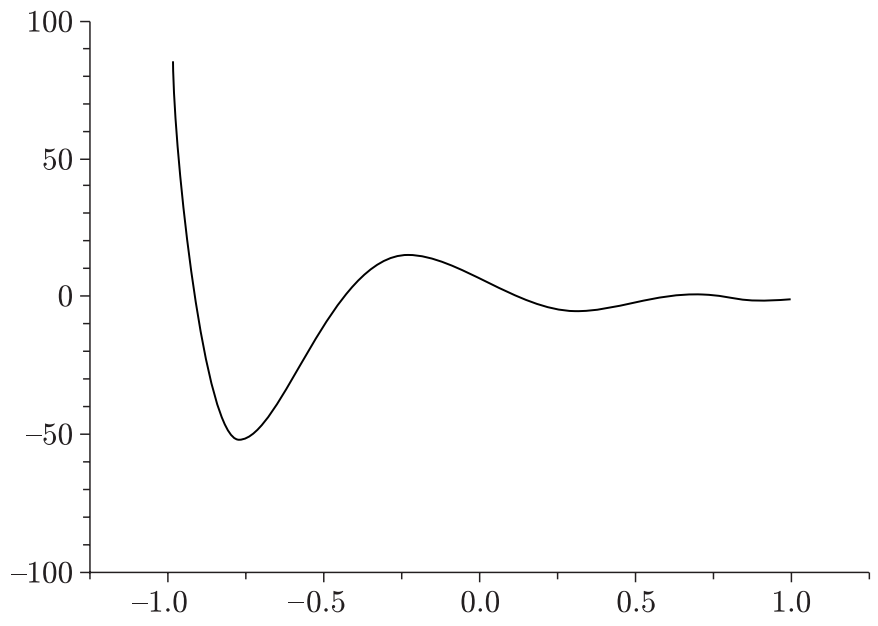

Рис. 17. Многочлен $\bar{C}_{4}(x, c, d)(2.25)$

Пусть $B$ - компактный самосопряженный положительный оператор, $\left(\gamma_{i}, \varphi_{i}\right)$ собственные пары оператора $B, \operatorname{Sp}(B) \in(0, M]$ (нуль, являющийся предельной точкой спектра, ему не принадлежит). Тогда оператор $B^{-1}$ сушествует и, являясь неограниченным, определен на всюду плотном в $H$ множестве $D\left(B^{-1}\right)$. Занумеруем точки $\gamma_{i} \in \operatorname{Sp}(B)$ в убываюшем порядке. Пусть $\eta, m$ - достаточно малые параметры регуляризации, $0 \leqslant \eta M \leqslant m<M$,

$$
A=(1-\eta) B+\eta M I \text {. }
$$

Тогда $\operatorname{Sp}(A) \in(\eta M, M], \lambda_{i}=(1-\eta) \gamma_{i}+\eta M$ и $D\left(A^{-1}\right)=H$. Подлежашее решению уравнение

$$
B u=f
$$

заменим уравнением (5.12). После такой замены для $f \in H$ будут сглажены коэффициенты в ряде Фурье, соответствующие малым значениям $\gamma_{i}$, значения которых, 
как правило, искажены ошибками в определении $f$. Пусть второй параметр регуляризации определяет отрезок $[m, M]$ для построения на нем ЧМБС-многочленов для того, чтобы с помощью начального приближения и параметров итерационного метода интенсивно определить те составляюшие в решении, которые соответствуют собственным значениям из $[m, M]$. В этом случае в ряде Фурье для $u$ основную роль будут играть, как правило, слагаемые, соответствующие собственным функциям с наибольшими собственными значениями. Сделав для заданного $m$ и $u^{0}=M^{-1} f n$ итераций по формулам (5.13), (5.19), мы видим, что приближение $u^{n}$ удовлетворяет уравнению (5.17). Оператор $A\left(I-P_{n}(A)(I-A / M)\right)^{-1}$ можно рассматривать при разумном выборе параметров итераций как регуляризатор и приближение к оператору $A$, нижняя граница спектра его равна $t=1 /\left(1 / M-P_{n}^{\prime}(0)\right)$, а собственные значения - $\lambda_{i} /\left(1-P_{n}\left(\lambda_{i}\right)\left(1-\lambda_{i} / M\right)\right)$. Второе уравнение в $(5.25)-$ исходное уравнение с измененной сглаженной правой частью $\left(I-P_{n}(A)(I-A / M)\right) f$, в которой прогрессивно с ростом $i$ уменьшены по абсолютной величине на множитель $\left(I-P_{n}\left(\lambda_{i}\right)\left(1-\lambda_{i} / M\right)\right) f_{i}$ коэффициенты Фурье $f_{i}=\left(f, \varphi_{i}\right)$ для $f$, соответствующие малым собственным значениям $\lambda_{i} \in[0, m]$.

Тогда согласно (5.17) $u^{n}$ является решением уравнений

$$
\begin{gathered}
A\left(I-P_{n}(A)(I-A / M)\right)^{-1} u^{n}=f, \text { или } A u^{n}=\left(I-P_{n}(A)(I-A / M)\right) f, \\
\varepsilon^{n}=P_{n}(A)(I-A / M) A^{-1} f .
\end{gathered}
$$

Эта формула показывает, что для определения параметров итерационного метода подходящими являются многочлены $C_{n}\left(x, \frac{1}{2}, \beta, C\right)$ при $\beta=0, \frac{1}{2}$ с весовой функцией $w(x)=(1+x)^{1 / 2}(1-x)^{\beta} /\left(1-\delta^{-1} x\right)$. Для лучшей регуляризации задачи $(7.2)$ целесообразно предварительно “отфильтровать” элемент $f$.

\section{§ 8. Взвешенные чебышёвские неявные операторные фильтры}

Цифровые фильтры широко применяются для обработки сигналов [42], [43] и для улучшения условий устойчивости явных методов решения задач математической физики [44], [45]. Пусть $\eta, \delta>0$ - достаточно малые числа, $L, D$ вместе с $D^{-1}-$ линейные ограниченные в $H$ операторы, $\left(\nu_{i}, \psi_{i}\right)$ - собственные пары оператора $L$ :

$$
L \psi_{i}=\nu_{i} \psi_{i}, \quad 0 \leqslant \nu_{i} \leqslant 1, \quad \nu_{i} \leqslant \nu_{i+1}
$$

$\psi_{i}$ образуют базис в $H$. Пусть $\widetilde{L}^{n}$ - расширение оператора $L^{n}, n \geqslant 1$, без учета граничных условий. Подлежаший фильтрации элемент $w \in H$ представим в виде $w=u+v$, где $D v \in \operatorname{ker} \widetilde{L}^{n}$. Считая $D v$ “гладким" элементом, фильтрации подвергнем лишь элемент $D u \in H$, для которого справедливо представление

$$
D u=\sum_{i} \bar{u}_{i} \psi_{i}
$$

где коэффициенты $\bar{u}_{i}$ зависят от выбора $v$. Поэтому, если это возможно, определим элемент $v$ так, чтобы частичные суммы в (8.2) достаточно быстро стремились к своему пределу. Это позволит ослабить после фильтрации эффект Гиббса. 
Если $P_{n}(t), n \geqslant 1,-$ многочлен вида (5.16), то элементу $u$ поставим в соответствие отфильтрованный элемент $D u^{F} \in H$ как решение уравнения

$$
\left(P_{n}(L)+\delta L^{n}\right) D u^{F}=P_{n}(L) D u .
$$

Тогда для коэффициентов $D u^{F}$ в представлении

$$
D u^{F}=\sum_{i} u_{i}^{F} \psi_{i}=\sum_{i} F_{n}\left(\nu_{i}\right) \bar{u}_{i} \psi_{i}
$$

функция $F(t)$, называемая переходной функиией, равна

$$
F_{n}(t)=\frac{1}{1+\delta t^{n} / P_{n}(t)}=\frac{1}{1+\delta / Q_{n}(1 / t)},
$$

где

$$
Q_{n}(z)=\prod_{i=1}^{n}\left(z-\gamma_{i}\right) ;
$$

при этом $F_{n}(0)=1, F_{n}^{(k)}(0)=0, k=1, \ldots, n-1$. Определим параметры $\gamma_{i}$ многочлена $P_{n}(t)$ так, чтобы функция $F_{n}(t)$ была мала в окрестности $t=1$. Точнее, пусть $F_{n}(1)=(-1)^{n} \eta$, тогда

$$
Q_{n}(1)=\frac{\eta \delta}{(-1)^{n}-\eta}
$$

Из (8.6) следует, что коэффициент при $z^{n}$ в $Q_{n}(z)$ равен единице. Определим из условий $(8.6),(8.7) Q_{n}(z)$, требуя, чтобы после замены переменной $z$ на $x=$ $(z-1-a) / a$ многочлен $Q_{n}(z)$ являлся приведенньм к отрезку $[1,1+2 a]$ ЧМБСмногочленом 1-го рода $C_{n}(x, 0,0, C)$ с весовой функцией $w(x)$. Из этих условий, учитывая (1.18) и значение $C_{n}(-1,0,0, C)$, определяем

$$
a=\left(\frac{\widetilde{A}_{n} \eta \delta}{\left(1-(-1)^{n} \eta\right) \sqrt{S(-1)}}\right)^{1 / n} .
$$

Тогда параметры $\gamma_{i}$ многочлена $P_{n}(t)$ равны

$$
\gamma_{i}=1+a\left(1-x_{i}\right), \quad i=1, \ldots, n,
$$

где $x_{i}$ - корни $(2.7)$ многочлена $C_{n}(x, 0,0, C)$. На рис. 18 приведен график $F_{3}(t)$ при $\eta=0.001, \delta=0.064, C_{3}(x, 0,0, C)=T_{3}(x)$. Заметим, что вид функции $F_{n}(t)$ не зависит от типа оператора $L$ и его собственных элементов $\psi_{i}$, но от них зависит разложение (8.2), а следовательно, и $D u^{F}$. Может возникнуть проблема с постановкой ненулевых граничных условий в уравнениях $(8.3),(8.4)$. Поэтому запишем уравнение для поправки $\Phi=D u-D u^{F}$ :

$$
\left(P_{n}(L)+\delta L^{n}\right) D \Phi=\delta L^{n} D u
$$

с однородньми краевьми условиями, требуя, чтобы $\Phi \equiv 0$ при $D u \in \operatorname{ker} \widetilde{L}^{n}$. Например, можно положить в некоторых дополнительных для $\Phi$ граничных узлах 


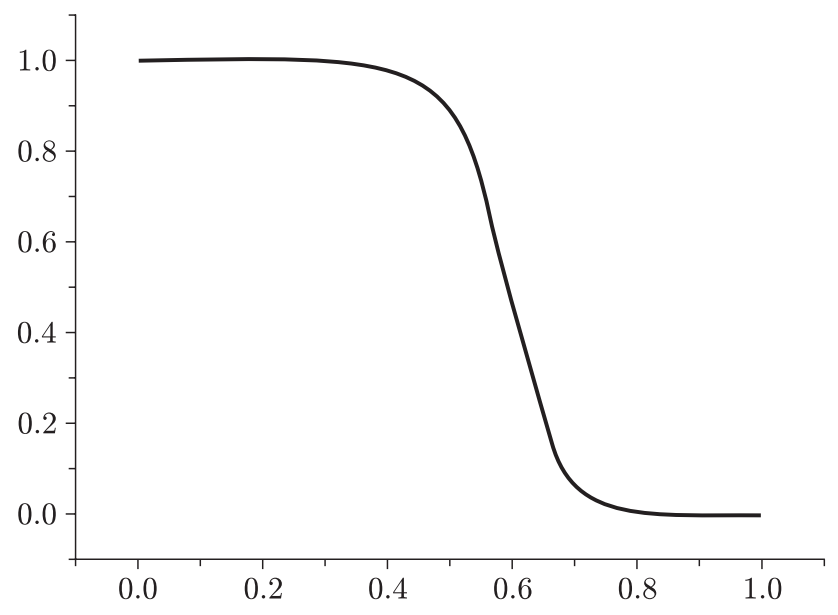

Рис. 18. Фильтр $F_{3}(t)(8.6)$

сетки $\Phi=0, L^{k} \Phi=0, k=1, \ldots, n-1$. Удачным выбором элемента $v$ можно значительно уменьшить краевой эффект Гиббса.

Простейшими примерами операторов $L$, заданных на внутренних узлах сетки, являются

$$
L_{ \pm} \Phi_{k}=0.5 \Phi_{k} \pm \frac{0.5\left(p_{k} \Phi_{k+1}+q_{k} \Phi_{k-1}\right)}{p_{k}+q_{k}}, k=1, \ldots, m, \quad \Phi_{0}=\Phi_{m+1}=0
$$

для одномерных сеток, аналогично

$$
L_{ \pm} \Phi_{k, j}=0.5 \Phi_{k, j} \pm \frac{0.125\left(p \Phi_{k, j+1}+q \Phi_{k, j-1}+r \Phi_{k+1, j}+s \Phi_{k-1, j}\right)}{p+q+r+s}
$$

для двумерных сеток, $p_{k}, q_{k}, p, q, r, s>0$, и т.д. $\left(L_{+}=I-L_{-}\right)$. Операторы типа $L_{-}$ориентированы на дифференциальные задачи математической физики, а $L_{+}$ - на сглаживания решений плохо обусловленных уравнений. Имея дополнительную информацию о классе подлежащих фильтрации функций, можно путем подбора коэффициентов в $L_{ \pm}$выбрать подходящую для этого класса систему собственных функций и тем самым улучшить [45] алгоритм фильтрации. Например, для одномерных задач, связанных с диффузией и конвекцией, характерньм для некоторого $b$ является оператор

$$
L_{b}=\frac{d^{2}}{d x^{2}}+b \frac{d}{d x}=\exp (-b x) \frac{d}{d x} \exp (b x) \frac{d}{d x} ;
$$

ему соответствует [44] разностньй на сетке $x_{k}=k h$ оператор

$$
\begin{aligned}
L_{-} \Phi_{k}= & 0.5 \Phi_{k}-0.25\left(\exp \left(\frac{b h}{2}\right) \Phi_{k+1}+\exp \left(-\frac{b h}{2}\right) \Phi_{k-1}\right) \\
& \times\left(\exp \left(\frac{b h}{2}\right)+\exp \left(-\frac{b h}{2}\right)\right)^{-1} .
\end{aligned}
$$


Собственные функции операторов $L_{b}, L_{ \pm}$имеют вид $\exp (-0.5 b x \pm i \omega x)$. Аналогичные формулы справедливы для двумерњых операторов. Отметим, что для одномерного случая в [44] изучены явные чебышёвские фильтры, а в [43] - неявные фильтры (8.4), (8.11) при

$$
L \Phi_{k}=L_{-} \Phi_{k}=0.5 \Phi_{k}-0.25\left(\Phi_{k-1}+\Phi_{k+1}\right), \quad k=1, \ldots, m,
$$

$P_{n}(L)=(I-L)^{n}, D=I$. В этом случае $\operatorname{ker} \widetilde{L}^{n}$ состоит из всех многочленов от $k g_{2 n-1}(k)$ до степени $2 n-1$ включительно. Тогда в представлении элемента $w=\left(w_{1}, \ldots, w_{m}\right)$ в виде $w_{k}=u_{k}+v_{k}$ положим $D v_{k}=g_{2 n-1}(k), k=1, \ldots, m$. Например, при $n=3$ полагаем

$$
g_{5}(k)=\frac{1}{2}\left(D w_{1}+D w_{m}+\left(D w_{1}-D w_{m}\right) y\left(x_{k}\right)\right), \quad k=1, \ldots, m,
$$

где

$$
y(x)=\frac{15}{8}\left(x-\frac{2}{3} x^{3}+\frac{1}{5} x^{5}\right), \quad x_{k}=\frac{m+1-2 k}{m-1}
$$

$\left(y( \pm 1)= \pm 1, y^{\prime}( \pm 1)=y^{\prime \prime}( \pm 1)=0\right)$. Тогда $u_{1}=u_{m}=0$.

Оператор $D$ в (8.3) для сеточных задач может быть реализован как деление на характерную так называемую форм-функцию.

\section{Список литературы}

1. Бахвалов Н. С., Кобельков Г. М., Жидков Н. П. Численные методы. М.-СПб.: Физматлит, 2000.

2. Пашковский $C$. Вычислительные применения многочленов и рядов Чебьшёва. М.: Наука, 1983.

3. Лебедев В. И. Функциональный анализ и вычислительная математика. М.: Физматлит, 2000.

4. Чебышёв П. Л. О функциях, мало удаляющихся от нуля при некоторых величинах переменных // Полное собрание сочинений. Т. 3. М.-Л.: ГИТТЛ, 1948. С. 108-120.

5. Марков A. А. Лекции о функциях, наименее уклоняющихся от нуля // Избранные труды. М.: ОГИЗ, 1948. С. 244-291.

6. Бернштейн C. Н. Об одном классе ортогональных многочленов // Собрание сочинений. Т. 1. М.: Изд-во АН СССР, 1952. С. 452-465.

7. Бернштейн С. Н. О многочленах, ортогоналњњых на конечном отрезке // Собрание сочинений. Т. 2. М.: Изд-во АН СССР, 1954. С. 7-106.

8. Szegö $G$. Über die Entwicrlung eine willkürlichen Funktion nach den Poynomen eines Orthogonalsystems // Math. Z. 1921. V. 12. P. 64-94.

9. Сегё Г. Ортогоналњые многочлены. М.: ГИФМЛ, 1962.

10. Лебедев В.И. О единой формуле для фазовой функции экстремальных ЧМБС-многочленов 1-4 рода // Докл. РАН. 2003. Т. 389. №1. С. 23-26.

11. Лебедев $В$. И. ЧМБС-многочлены и квадратурные формулы повьшенной точности для некоторых классов интегралов с зависящими от параметров весовьми функциями // Докл. РАН. 2003. Т. 390. № 5. С. 1-5.

12. Лебедев В. И. ЧМБС-многочлены и алгоритмы оптимизации чебьшёвских итерационных методов на классах начальных ошибок // Докл. РАН. 2003. Т. 393. №3. С. 1-5.

13. Лебедев В. И. Экстремальные ЧМБС-многочлены 1-4 рода и методы оптимизации вычислительых алгоритмов // Труды матем. ин-та им. Н.И. Лобачевского. Т. 20. Казань: Казанское матем. общ-во, 2003. С. 37-86.

14. Лебедев В.И. Итерационные методы решения линейных операторных уравнений и многочлены, наименее отклоняющиеся от нуля // Математический анализ и смежные вопросы математики. Новосибирск: Наука, 1978. С. 89-108. 
15. Lebedev V.I. Extremal polynomials with restrictions and optimal algorithms // Advanced mathematics: computations and applications. Novosibirsk: NCC Publisher, 1995. P. 491-502.

16. Lebedev V.I. A new method for determining the roots of polynomials of least deviation on a segment with weight and subject to additional conditions. Part I, Part II // Russian J. Numer. Anal. Math. Modelling. 1993. V. 8. № 3. P. 195-222; 1993. V. 8. № 5. P. 397-426.

17. Марчук Г.И., Лебедев В.И. Численные методы в теории переноса нейтронов. М.: Атомиздат, 1981.

18. Крейн М. Г., Нудельман А. А. Проблема моментов Маркова и экстремалњные задачи. М.: Наука, 1973.

19. Ахиезер Н. И. Лекции по теории аппроксимации. М.: Наука, 1965.

20. Геронимус $Я$. Л. О некоторых уравнениях в конечных разностях и соответствующих системах ортогональыых полиномов // Докл. АН СССР. 1940. Т. 29. С. 536-538.

21. Геронимус $A$. Л. О положительных тригонометрических полиномах и гармонических функциях // Докл. АН СССР. 1946. Т. 51. С. 569-572.

22. Геронимус $Я$. Л. О некоторых уравнениях в конечных разностях и соответствующих системах ортогональных многочленов // Записки матем. отд. физ.-матем. ф-та ХТУ и Харьковского матем. общества. 1957. Т. XXV. № 4. С. 87-100.

23. Лебедев В.И. О решении обратных задач и тригонометрических формах для многочленов Геронимуса // Russian J. Num. Anal. Math. Modelling. 2000. T. 15. № 1. С. 73-93.

24. Лебедев В.И. Об итерационных методах решения операторных уравнений, спектр которых лежит на нескольких отрезках // ЖВМ и МФ. 1969. Т. 9. №6. С. 1247-1253.

25. Lebedev V.I. Chebyshev and optimals-in-mean iterative methods for problems with spectrum contained in two segments or incidelemniscates // East-West. J. Numer. Math. 1994. V. 2. № 2. P. 107-127.

26. Lebedev V.I., Zabelin V. V. On one class of three-term iterative methods with Chebyshev parameters // Sov. J. Numer. Anal. Math. Modelling. 1990. V. 5. № 4/5. P. 275-298.

27. Натансон И. П. Конструктивная теория функций. М.-Л.: ГИТТЛ, 1949.

28. Крылов В. И. Приближенное выгисление интегралов. М.: ГИФМЛ, 1959.

29. Мысовских И. П. Лекции по методам вычислений. СПб.: Изд-во СПбГУ, 1998.

30. Бабурин О.В., Лебедев В.И. О вычислении интегралов в смысле главного значения весов и узлов квадратурных формул Гаусса // ЖВМи МФ. 1965. Т. 5. № 3. С. 451-462.

31. Бабурин О. В., Лебедев В. И. О вьчислении таблиц корней и весов полиномов Эрмита и Лагерра для $n=1(1) 101$ // ЖВМ и МФ. 1967. Т. 7. № 4. С. 1021-1030.

32. Лебедев В. И. Алгоритмы определения параметров одного класса квадратурных формул // Вопросы вычислительной и прикладной математики. № 51. Ташкент: Изд-во АН Узб. ССР, 1978. С. 44-51.

33. Лебедев В. И. Квадратурные формулы по поверхности вытянутого эллипсоида вращения // Кубатурные формулы и их приложения. Улан-Удэ: Изд-во Вост.-Сиб. гос. тех. ун-та, 1997. С. 48-56.

34. Градштейн И. С., Рыжик И. М. Таблицы интегралов, сумм, рядов и произведений. М.: Наука, 1971.

35. Никольский С. М. Квадратурные формулы. М.: Наука, 1988.

36. Довгий С. А., Лифанов И. К. Методы решения интегральных уравнений. Киев: Наукова думка, 2002.

37. Бернштейн C. Н. Об асимптотическом значении наилучшего приближения аналитических функций // Собрание сочинений. Т. І. М.: Изд-во АН СССР, 1952. С. 127-135.

38. Гавурин $M . K$. Применение полиномов наилучшего приближения к улучшению сходимости итеративных процессов // УМН. 1950. Т. 5. №3(37). С. 156-160.

39. Федоренко Р. П. Введение в вычислительну физику. М.: Изд-во МФТИ, 1994.

40. Шайдуров В. В. Многосеточные методы конечных элементов. М.: Наука, 1989.

41. Тихонов A. Н., Арсенин B. Я. Методы решения некорректных задач. М.: Наука, 1986.

42. Хемминг Р. В. Цифровые фильтры. М.: Наука, 1987. 
43. Wiliam H. R. High-order low-pass implicit tangent filters for use in finite area calculations // Monthly Weather Review. 1988. V. 116. P. 2132-2141.

44. Лебедев В. И. Явные разностные схемы для решения жестких задач с комплексным или разделимым спектром // ЖКВМ и МФ. 2000. Т. 40. № 12. С. 1801-1812.

45. Lebedev V.I. Extremal polynomials, pseudo-difference operators, and stable explicit schemes for the solution of stiff nonstationary problems of mathematical physics // Proc. of the International Conference on Computational Mathematics. Part I. Novosibirsk, 2002. P. 60-63.

РНЦ "Курчатовский институт",

Институт вычислительной математики РАН, г. Москва

Поступила в редакцию

E-mail: nucrect@inm.ras.ru

26.02 .2004

lebedev@vver.kiae.ru 